\title{
苯基吡咯类杀菌剂的设计合成及三维-定量构效关系(3D-QSAR)研究
}

\author{
$\begin{array}{cccccc}\text { 徐洪亮 } a & \begin{array}{llll}\text { 苏静 } \\ \end{array} & \text { 王子时*,a } & \text { 侯晨忻 } a & \text { 吴鹏冲 } a & \text { 邢 } a \\ & \text { 李香帅 } a & \text { 朱晓磊 } b & \text { 路运才*,a } & \text { 徐利剑 } a & \end{array}$ \\ ( $a$ 黑龙江大学现代农业与生态环境学院 哈尔滨 150080) \\ ( $b$ 华中师范大学化学学院 武汉 430070)
}

\begin{abstract}
摘要 为寻找新型吡咯类农药，基于杀菌剂氟咯菌腈设计合成了 21 个苯基吡咯类化合物，在吡咯环上引入甲基基团， 其目的是探究 $\mathrm{N}$ 位取代基对该类化合物活性的影响. 通过 ${ }^{1} \mathrm{H}$ NMR、FTIR、单晶 X 射线衍射、高分辨质谱、元素分析 和熔点测定等对目标化合物结构进行了表征与确认，并通过挥发法培养得到 16 个目标化合物的单晶结构. 5 种病原菌 抑菌活性测试结果显示：在 $10 \mathrm{mg} / \mathrm{L}$ 浓度条件下，4-(2-氯苯基)-1H-吡咯-3-腈(4b), 4-(2-溴苯基)-1H-吡咯-3-腈(4c), 4-(2-(三氟甲基)苯基)-1 $H$-吡咯-3-腈(4d), 4-(2-氯-3-氟苯基)- $1 H$-吡咯-3-腈(4g), 4-(2,3-二氯苯基)- $1 H$-吡咯-3-腈(4h)对 4 种 病菌表现出较好甚至高于阳性对照的抑菌效果，其中化合物 $\mathbf{4 g}$ 在 $1 \mathrm{mg} / \mathrm{L}$ 浓度条件下对 3 种病菌的抑制效果仍达到 $80 \%$ 以上，而氮位甲基取代的目标化合物对水稻纹枯病菌表现出专一的抑菌活性. 为了开发出更有效的抗水稻纹枯病菌化 合物, 采用比较分子力场分析(CoMFA)方法对 20 个化合物的水稻纹枯病菌活性进行初步的三维一定量构效关系 (3D-QSAR)研究, 建立了一个有效的 CoMFA 模型 $\left(q^{2}=0.503, r^{2}=0.974\right)$, 展现了良好的预测能力, 为后续该系列化合物 的进一步优化提供了理论支持.
\end{abstract}

关键词 吡咯类化合物; 杀菌剂; 氮位取代; 生物活性; 三维-定量构效关系(3D-QSAR)

\section{Synthesis, Design and Three-Dimensional Quantitative Structure Activity Relationship (3D-QSAR) Research of Phenylpyrrole Fungicides}

\author{
Xu, Hongliang ${ }^{a}$ \\ $\mathrm{Su}$, Jing $^{a}$ \\ Wang, Zishi ${ }^{*}, a$ \\ Hou, Chenxin ${ }^{a}$ \\ Wu, Pengchong ${ }^{a}$ \\ Xing, Yue ${ }^{a}$ \\ Li, Xiangshuai ${ }^{a}$ \\ Zhu, Xiaolei ${ }^{b}$ \\ Lu, Yuncai*,a \\ $\mathrm{Xu}, \mathrm{Lijian}^{a}$ \\ $\left({ }^{a}\right.$ College of Modern Agriculture and Ecological Environment, Heilongjiang University, Harbin 150080) \\ $\left({ }^{b}\right.$ College of Chemistry, Central China Normal University, Wuhan 430079)
}

\begin{abstract}
In order to find new pyrrole pesticides, twenty one new pyrrole compounds based on the structure of fludioxonil were designed and synthesized. And a methyl group was introduced into the pyrrole ring to explore the effect of N-position substituent on the activity. All the structures of target products and intermediates were characterized and confirmed by ${ }^{1} \mathrm{H}$ NMR, FTIR, and single crystal X-ray diffraction, HRMS, elemental analysis and melting point determination. The single crystals of sixteen final products were obtained by volatilizing at room temperature. The antibacterial activity test results on the five pathogens show that under the concentration of $10 \mathrm{mg} / \mathrm{L}, 4-(2$-chlorophenyl)- $1 H$-pyrrole-3-carbonitrile (4b), 4-(2-bromopheny)-1H-pyrrole-3-carbonitrile (4c), 4-(2-(trifluoromethyl)phenyl)-1H-pyrrole-3-carbonitrile (4d), 4-(2-chloro-3-fluorophenyl)-1H-pyrrole-3-carbonitrile (4g), and 4-(2,3-dichlorophenyl)-1H-pyrrole-3-carbonitrile (4h) showed even better or higher antibacterial effect on the four pathogens, the inhibitory effect of compound $\mathbf{4 g}$ on the three pathogens at a concentration of 1
\end{abstract}

\footnotetext{
* Corresponding authors. E-mail: 2016048@hlju.edu.cn; luyuncai@hlju.edu.cn

Received March 24, 2021; revised June 14, 2021; published online July 12, 2021.

Project supported by the National Key Research and Development Program of China (No. 2017YFD0300405), the Natural Science Foundation of Heilongjiang Province (Nos. LH2019C055, LH2019C056), the Project Funded by China Postdoctoral Science Foundation (No. 2019M651316), the Heilongjiang Provincial Postdoctoral Science Foundation (No. LBH-Z18261), the Department of Education of Heilongjiang Province (Nos. 2020-KYYWF-1029, 2020-KYYWF-1030, 2020-KYYWF-1031, 135409216), and the Heilongjiang University Graduate Innovative Research Fund Project (No. YJSCX2020-080HLJU).

国家重点研发计划(No. 2017YFD0300405)、黑龙江省自然科学基金(Nos. LH2019C055, LH2019C056)、中国博士后科学基金(No. 2019M651316)、黑 龙江省博士后科学基金(No. LBH-Z18261)、黑龙江省省属高校基本科研(Nos. 2020-KYYWF-1029, 2020-KYYWF-1030，2020-KYYWF-1031, 135409216)、黑龙江大学研究生创新科研重点(No. YJSCX2020-080HLJU)资助项目.
} 
$\mathrm{mg} / \mathrm{L}$ also reached more than $80 \%$. The target compounds substituted with methyl at the nitrogen position showed specific antibacterial activity against Rhizoctonia solani. In order to develop more effective antifungal compounds against Rhizoctonia solani, the three-dimensional quantitative structure activity relationship (3D-QSAR) study on the activity of 20 compounds against Rhizoctonia solani was preliminary conducted using comparative molecular force field analysis (CoMFA). A CoMFA model $\left(q^{2}=0.503, r^{2}=0.974\right)$ was established, which showed good predictive ability, and also provided theoretical support for the further optimization of this series of compounds.

Keywords pyrrole compounds; fungicide; nitrogen substitution; biological activity; three-dimensional quantitative structure activity relationship (3D-QSAR)

吡咯作为一类重要的五元含氮杂环结构 ${ }^{[1-3]}$, 近年 来在化工、医药和半导体材料 ${ }^{[4-5]}$ 等行业有着广泛的用 途. 在农药领域相较其他含氮杂环结构, 如吡唑、三唑、 吡啶、嘧啶等, 吡咯类化合物的农药品种 ${ }^{[6-7]}$ 相对较少, 这给予科研工作者更广阔的研究空间. 目前在市场中具 有代表性的吡咯类农药品种有杀菌剂拌种咯、氟咯菌 腈[8-10]、杀虫剂澳虫腈[11-12]、除草剂氟咯草酮[13]等, 其 中值得关注的是由先正达公司开发的新型吡咯类杀菌 剂氟咯菌腈, 其具有高效、广谱、用量少、持效长等优 点, 一经上市便得到了广泛关注, 是全球销量最大的种 子处理剂之一.

氟咯菌腈主要是以 2,2-二氟胡椒环-4-甲醛 ${ }^{[14]}$ 作为 起始原料, 通过缩合反应、加成反应等进行制备, 存在 合成原料成本高、自行合成难度大等问题. 从结构上来 说其主要活性基团为苯环上取代的氟原子和氧基吡咯 结构 ${ }^{[15]}$, 本课题组前期基于此对氟咯菌腈的主体结构 进行修饰, 在保留其氰基吡咯结构的基础上, 引入同样 具有较好生物活性的五元杂环吡唑环, 合成了一系列吡 唑联吡咯类化合物 ${ }^{[16]}$, 以期提高其生物活性. 测试结果 显示该类化合物虽表现出一定的生物活性, 但与氟咯菌 腈还存在着较大差距.

氟咯菌腈是通过天然产物的结构改造先得到杀菌 剂拌种咯, 再通过结构修饰进而开发出来的, 我们发现 拌种咯的起始原料是二取代的苯甲醛, 较氟咯菌腈的起 始原料结构简单. 选择此类结构作为原料, 使其合成成 本和合成难度大大降低, 合成步骤简化, 更是降低了环 保风险. 以此为基础对氟咯菌腈结构进一步修饰, 选取 一取代或多取代的苯甲醛作为起始原料进行合成. 含氮 杂环的 $\mathrm{N}$ 位以甲基基团或芳环基团取代, 开发出了许多 农药杀菌剂, 例如唑菌脂、唑胺菌脂、氟唑菌酰胺

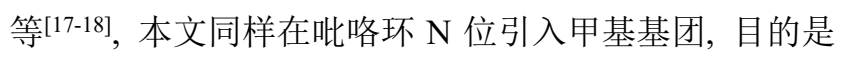
探究 $\mathrm{N}$ 位取代基对该类化合物生物活性的影响. 最终合 成了 21 个苯基吡咯类化合物, 通过 ${ }^{1} \mathrm{H}$ NMR、FTIR、单 晶 $\mathrm{X}$ 射线衍射、高分辨质谱、元素分析和熔点测定等测 试手段, 对目标化合物结构进行了表征与确认, 并通过 挥发法培养得到 16 个目标化合物的单晶结构. 抑菌活 性测试结果表明, 其中 5 个化合物对 4 种病菌表现出较 好甚至高于阳性对照的抑菌效果. 此外, 对目标化合物
的水稻纹枯病菌活性进行初步的三维-定量构效关系 (3D-QSAR)研究 ${ }^{[19]}$, 建立了一个有效的 CoMFA 模型, 为后续该类化合物的研究与开发提供了理论支持.

\section{1 结果与讨论}

\section{1 目标化合物的合成}

目标化合物的合成路线如 Scheme 1 所示. 以一取 代或二取代的苯甲醛为原料, 在碱性条件下通过 Knoevenagel 缩合反应得到中间体 2 (2-㲵基丙烯酰胺类 化合物), 选择乙醇作为溶剂进行回流反应, 相较于文 献[16]中以三乙胺作为碱的方法, 本工作选取醋酸铵和

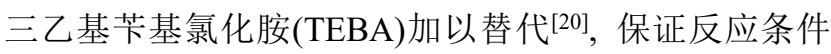
温和，后处理方便；中间体 3 (TosMIC-对甲苯磺酰甲基 异腈)是合成五元杂环重要的中间体之一, 广泛应用在 医药、农药和染料等各个领域, 现有报道的关于 TosMIC 合成方式有两种, 为一锅法和分步法, 本工作选择分步 法进行合成保证了每一步反应的收率和纯度能达到最 佳效果，也是本课题组前期实验总结得到的方法之一; 目标化合物 $\mathbf{4}$ 是由中间体 $\mathbf{2}$ 和中间体 $\mathbf{3}$ 通过加成反应和 关环反应得到的，首先是在碱性条件使中间体 3 亚甲基 上的碳原子形成碳负离子, 然后在碳碳双键上发生 Michael 加成反应, 再进行关环脱去 $\mathrm{TsOH}$ 得到目标化合 物 4, 该反应选取甲醇-二氯甲烷混合溶液作为溶剂, 使 反应物更好地溶于溶剂中, 既提高了反应收率, 又可以 进行部分回收降低溶剂成本 ${ }^{[21]}$; 目标化合物 5 是由碘甲 烷(甲基化试剂)和目标化合物 4 在碱性条件下发生亲核 取代反应得到的 ${ }^{[22-23]}$, 碘甲烷较其他甲基化试剂反应速 率更快、用量更少, 选取物料配比为目标化合物 4 : 碳 酸钾：碘甲烷 $=1: 1.2: 1.5$ 会有效的缩短反应时间并 提高收率. 目标化合物 $\mathbf{4 a} \sim \mathbf{4 k}, \mathbf{5 a} \sim \mathbf{5 j}$ 的结构如表 1 所 示.

\section{2 目标化合物的表征}

\subsection{1 红外谱图分析}

红外谱图显示, 目标化合物 $\mathbf{4 a} \sim \mathbf{4 k}, 5 \mathbf{a} \sim \mathbf{5 j}$ 均在 $2200 \sim 2260 \mathrm{~cm}^{-1}$ 处有明显的特征吸收, 证明是 $\mathrm{C} \equiv \mathrm{N}$ 的 伸缩振动; 在 $1500 \sim 1600 \mathrm{~cm}^{-1}$ 处的特征峰则是 $\mathrm{C}=\mathrm{C}$ 双键的伸缩振动; 在目标化合物 $\mathbf{4 a} \sim \mathbf{4 k}$ 的结构中存在 
着 $\mathrm{N}-\mathrm{H}$ 键的伸缩振动, 均出现在 $3200 \sim 3300 \mathrm{~cm}^{-1}$ 处, 而目标化合物 5a $\sim \mathbf{5 j}$ 则在 $3200 \sim 3300 \mathrm{~cm}^{-1}$ 处没有出现 明显的特征峰, 在 $1100 \sim 1200 \mathrm{~cm}^{-1}$ 处出现一个明显的 特征峰, 即 $\mathrm{N}-\mathrm{CH}_{3}$ 键伸缩振动, 这证明吡咯环上原有 的 $\mathrm{H}$ 原子是被甲基所取代, 从而产生这样的特征峰变 化.
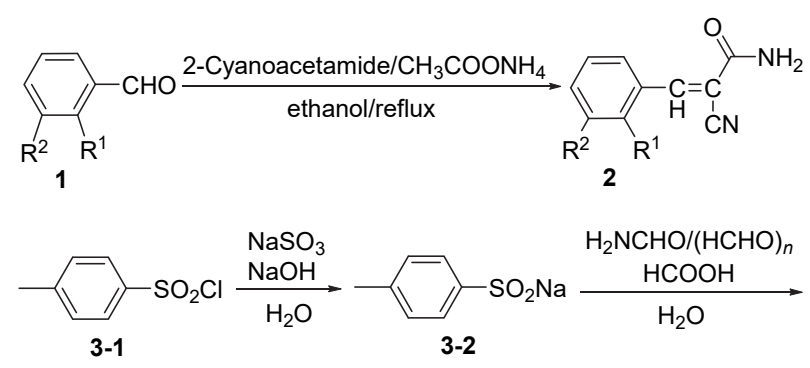

$\mathrm{OCl}_{3}$

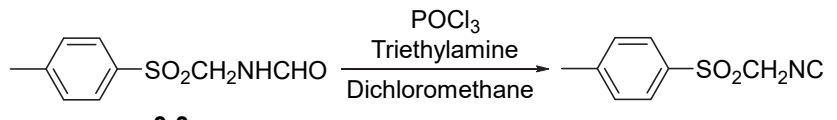

3-3

3

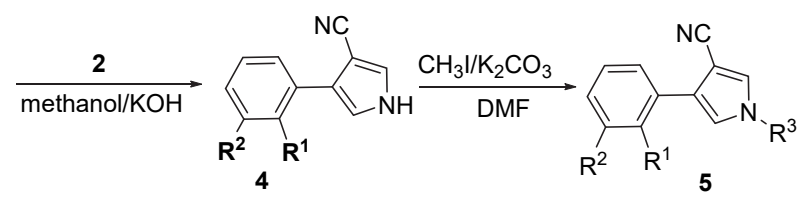

$\mathrm{R}^{1}=\mathrm{F}, \mathrm{Cl}, \mathrm{Br}, \mathrm{CF}_{3}, \mathrm{Me}, \mathrm{OCH}_{3}$

$\mathrm{R}^{2}=\mathrm{H}, \mathrm{F}, \mathrm{Cl}, \mathrm{CF}_{3}, \mathrm{Me}, \mathrm{OCH}_{3}$

$\mathrm{R}^{3}=\mathrm{H}, \mathrm{Me}$

图式 1 苯基吡咯类化合物合成路线

Scheme 1 Synthetic route of phenylpyrrole compounds

表 1 目标化合物结构

Table 1 Structures of the target compounds

\begin{tabular}{|c|c|c|c|}
\hline Compd. & $\mathrm{R}^{1}$ & $\mathrm{R}^{2}$ & $\mathrm{R}^{3}$ \\
\hline $4 a$ & $\mathrm{~F}$ & $\mathrm{H}$ & $\mathrm{H}$ \\
\hline $4 b$ & $\mathrm{Cl}$ & $\mathrm{H}$ & $\mathrm{H}$ \\
\hline $4 c$ & $\mathrm{Br}$ & $\mathrm{H}$ & $\mathrm{H}$ \\
\hline 4d & $\mathrm{CF}_{3}$ & $\mathrm{H}$ & $\mathrm{H}$ \\
\hline $4 e$ & $\mathrm{CH}_{3}$ & $\mathrm{H}$ & $\mathrm{H}$ \\
\hline $4 f$ & $\mathrm{OCH}_{3}$ & $\mathrm{H}$ & $\mathrm{H}$ \\
\hline $4 g$ & $\mathrm{Cl}$ & $\mathrm{F}$ & $\mathrm{H}$ \\
\hline $4 h$ & $\mathrm{Cl}$ & $\mathrm{Cl}$ & $\mathrm{H}$ \\
\hline $4 i$ & $\mathrm{CH}_{3}$ & $\mathrm{CH}_{3}$ & $\mathrm{H}$ \\
\hline $4 j$ & $\mathrm{OCH}_{3}$ & $\mathrm{OCH}_{3}$ & $\mathrm{H}$ \\
\hline $4 k$ & $\mathrm{~F}$ & $\mathrm{CF}_{3}$ & $\mathrm{H}$ \\
\hline $5 a$ & $\mathrm{~F}$ & $\mathrm{H}$ & $\mathrm{CH}_{3}$ \\
\hline $5 b$ & $\mathrm{Cl}$ & $\mathrm{H}$ & $\mathrm{CH}_{3}$ \\
\hline $5 c$ & $\mathrm{Br}$ & $\mathrm{H}$ & $\mathrm{CH}_{3}$ \\
\hline $5 d$ & $\mathrm{CF}_{3}$ & $\mathrm{H}$ & $\mathrm{CH}_{3}$ \\
\hline $5 e$ & $\mathrm{CH}_{3}$ & $\mathrm{H}$ & $\mathrm{CH}_{3}$ \\
\hline $5 f$ & $\mathrm{OCH}_{3}$ & $\mathrm{H}$ & $\mathrm{CH}_{3}$ \\
\hline $5 \mathrm{~g}$ & $\mathrm{Cl}$ & $\mathrm{F}$ & $\mathrm{CH}_{3}$ \\
\hline $5 \mathrm{~h}$ & $\mathrm{Cl}$ & $\mathrm{Cl}$ & $\mathrm{CH}_{3}$ \\
\hline $5 i$ & $\mathrm{CH}_{3}$ & $\mathrm{CH}_{3}$ & $\mathrm{CH}_{3}$ \\
\hline $5 \mathrm{j}$ & $\mathrm{OCH}_{3}$ & $\mathrm{OCH}_{3}$ & $\mathrm{CH}_{3}$ \\
\hline
\end{tabular}

\subsection{2 核磁谱图分析}

${ }^{1} \mathrm{H}$ NMR 谱图显示, 目标化合物 $\mathbf{4 a} \sim \mathbf{4 k}, \mathbf{5 a} \sim \mathbf{5 j}$ 苯 环上的 $\mathrm{H}$ 化学位移在 $\delta 7 \sim 8$ 之间, 苯环上 $\mathrm{CH}_{3}$ 取代的 $\mathrm{H}$ 化学位移在 $\delta 2 \sim 2.5$ 之间, 同时目标化合物 $\mathbf{4 a} \sim \mathbf{4 k}$ 在化 学位移 $\delta 11.8 \sim 12.1$ 之间均出现一个明显的单峰, 即吡 咯环氮上的 $\mathrm{H}$, 而目标化合物 $\mathbf{5 a} \sim \mathbf{5} \mathbf{j}$ 在化学位移 $\delta$ $3.69 \sim 3.73$ 之间出现一个明显的单峰, 即甲基上的 $\mathrm{H}$, 再次证明吡咯环上原有的 $\mathrm{H}$ 原子是被甲基所取代.

${ }^{13} \mathrm{C} \mathrm{NMR}$ 谱图显示, 目标化合物 $\mathbf{4 a} \sim \mathbf{4 k}, \mathbf{5 a} \sim \mathbf{5 j}$ 苯 环上和吡咯环上的 C 化学位移在 $\delta 110 \sim 130$ 之间, 苯环 上取代基的不同，导致 $\mathrm{C}$ 原子化学位移出现明显变化, 当取代基为甲基、甲氧基等供电子基团时，化学位移则 向高场方向移动，取代基为 $\mathrm{Cl} 、 \mathrm{Br}$ 等吸电子基团时则变 化较小; 而取代基为 $\mathrm{F}$ 原子时, 则出现了偶合现象, 最 明显的就是苯环上与 $\mathrm{F}$ 原子相连的 $\mathrm{C}$ 原子，偶合常数一 般在 $245 \mathrm{~Hz}$ 左右, 在核磁谱图中可看见化学位移在 $\delta$ 155 158 之间的双重峰, 同时化学位移在 $\delta \quad 120 \sim 127$ 之间同样还会出现不同程度的偶合, 谱图中呈现出的偶 合裂分现象也表明化合物结构中确实有 $\mathrm{F}$ 原子存在; 另 外目标化合物 $\mathbf{5 a} \sim \mathbf{5 j}$ 的谱图中, 化学位移在 $\delta 36.5$ 左右 出现了一个峰, 为引入甲基上的 $\mathrm{C}$ 原子化学位移, 再次 证明发生了甲基化反应，这与核磁共振氢谱的结论相吻 合.

\subsection{3目标化合物晶体结构分析}

以目标化合物 $4 \mathrm{~d}$ (晶体结构数据存于剑桥晶体数据 库, CCDC: 2075823)为例, 将样品充分溶解在乙醇和水 $(V: V=80: 20)$ 的混合溶剂中. 通过自然挥发法得到无 色透明的晶状固体，通过单晶 $\mathrm{X}$ 射线衍射分析得到目标 化合物 $4 \mathbf{d}$ 的分子式为 $\mathrm{C}_{12} \mathrm{H}_{7} \mathrm{~F}_{3} \mathrm{~N}_{2}$, 分子量 $M_{\mathrm{r}}=236.20$, Triclinic, $P-1$ 空间群. 晶胞参数: $a=9.2418(6) \mathrm{nm}, b=$ 10.7743(7) nm, $c=12.0462(7) \mathrm{nm} ; \alpha=72.010(2)^{\circ}, \beta=$ $73.121(2)^{\circ}, \gamma=88.204(3)^{\circ}, V=1.08945(12) \mathrm{nm}^{3} ; D_{x}=$ $1.440 \mathrm{~g} / \mathrm{cm}^{3} ; Z=4 ; F(000)=480.0 ; \mu=0.123 \mathrm{~mm}^{-1}$. 晶体 结构修正后的一致性因子 $R=0.0485, w R=0.1150$. 其不 对称单元图可以很好地表征该化合物的分子结构, 如图 1 所示.

化合物 $4 \mathrm{c} 、 4 \mathrm{e} \sim 4 \mathrm{f} 、 4 \mathrm{~h} \sim \mathbf{4 k}$ 的分子结构与 $4 \mathrm{~d}$ 相似， 其具体的单晶数据参数、 $\mathrm{CCDC}$ 编号以及单分子结构图 在辅助材料中有详细说明.

以目标化合物 $\mathbf{5 g}$ (晶体结构数据存于剑桥晶体数据 库, CCDC: 2075859)为例, 将样品充分溶解在乙醇和水 $(80: 20, V: V)$ 的混合溶剂中, 通过自然挥发法得到无 色透明的晶状固体，通过单晶 $\mathrm{X}$ 射线衍射分析，目标化 合物 $5 \mathrm{~g}$ 的分子式为 $\mathrm{C}_{12} \mathrm{H}_{8} \mathrm{ClFN}_{2}$, 分子量 $M_{\mathrm{r}}=234.65$, 单斜晶系, $P 2_{1} / n$ 空间群. 晶胞参数: $a=3.89690(10) \mathrm{nm}$, 


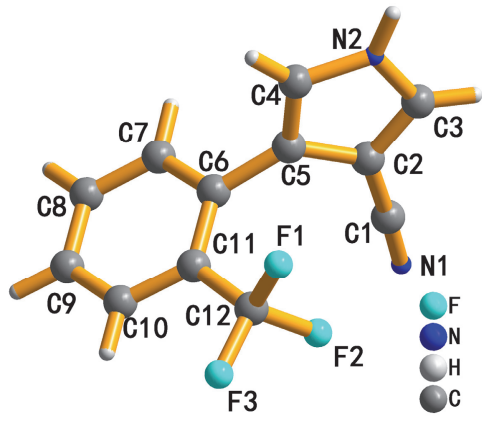

图 $14 \mathrm{~d}$ 的单分子结构

Figure 1 Single molecule structure of compound $\mathbf{4 d}$

$b=23.4679(8) \mathrm{nm}, c=11.6201(4) \mathrm{nm} ; \alpha=90^{\circ}, \beta=$ $92.1530(10)^{\circ}, \gamma=90^{\circ}, V=1.06193(6) \mathrm{nm}^{3} ; D_{x}=1.468$ $\mathrm{g} / \mathrm{cm}^{3} ; Z=4 ; F(000)=480.0 ; \mu=0.343 \mathrm{~mm}^{-1}$. 晶体结构 修正后的一致性因子 $R=0.0483, w R=0.1304$. 其不对称 单元图可以很好地表征该化合物的分子结构, 如图 2 所 示.

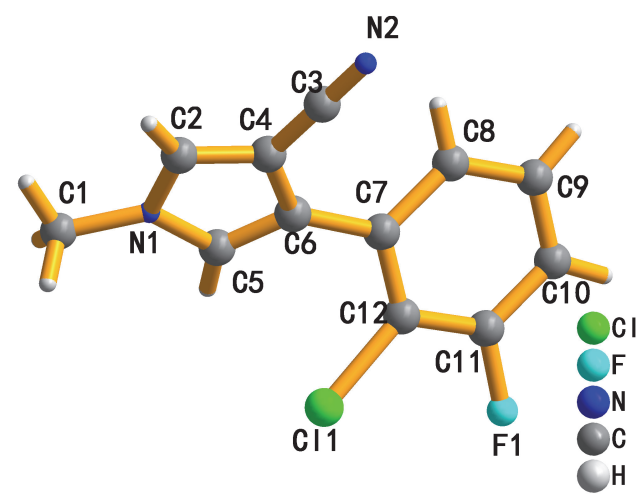

图 $25 \mathrm{~g}$ 的单分子结构

Figure 2 Single molecule structure of compound $\mathbf{5 g}$

化合物 $5 \mathrm{a} 、 5 \mathrm{c} \sim 5 \mathrm{f} 、 5 \mathrm{i} \sim 5 \mathrm{j}$ 的结构与 $5 \mathrm{~g}$ 相似, 具体 的单晶数据参数、 $\mathrm{CCDC}$ 编号以及单分子结构图在辅助 材料中有详细说明.

以目标化合物 $4 \mathbf{d}$ 和 $\mathbf{5 d}$ 为例, 通过单晶结构可以看 出 $4 d$ 的 $\mathrm{C}-\mathrm{C}$ 键键长在 0.1364(3) 0.1493(3) nm, C- $\mathrm{F}$ 键键长在 $0.1330(2) \sim 0.1338(3) \mathrm{nm}$ 之间, 吡咯环上的 $\mathrm{C}-\mathrm{N}$ 键键长在 0.1337(2) 0.1367(2) $\mathrm{nm}$ 之间, $\mathrm{C} \equiv \mathrm{N}$ 键 键长为 0.1144(3) nm, 除 $\mathrm{C} \equiv \mathrm{N}$ 键之外的其他键角在 $104.84^{\circ} \sim 127.71^{\circ}$ 之间; $5 \mathbf{d}$ 的 $\mathrm{C}-\mathrm{C}$ 键键长在 0.1368(2) $0.1496(3) \mathrm{nm}$ 之间, $\mathrm{C}-\mathrm{F}$ 键键长在 0.1318(3) 0.1344(3) $\mathrm{nm}$ 之间, 吡咯环上的 $\mathrm{C}-\mathrm{N}$ 键键长在 0.1349(2) $0.1366(2) \mathrm{nm}$ 之间, $\mathrm{C} \equiv \mathrm{N}$ 键键长为 $0.1144(2) \mathrm{nm}$, 除 $\mathrm{C} \equiv \mathrm{N}$ 键之外的其他键角在 $104.6^{\circ} \sim 127.65^{\circ}$ 之间. 比较 数据发现, 在吡咯环氮位引入甲基基团后各原子间键长 键角均未出现明显改变, 说明烷基取代基对该类化合物 结构影响不大, 其活性没有明显提高, 这与后续活性测 试结果相吻合; 但由于氮位 $\mathrm{H}$ 原子被取代后, 导致整体
结构取代位点减少、结合氢键能力变差、结构更加稳定, 因此表现出活性有所减弱但抑菌效果专一的特点.

\section{3 抑菌活性测试}

测试化合物对水稻恶苗病菌 (Fusarium moniliforme)、水稻纹枯病菌(Rhizoctonia solani)、稻瘟病菌 (Pyricularia oryzae)、黄瓜枯萎病菌(Fusarium oxysporum f.sp. cucumerinum) 和大豆根腐病菌 (Fusarium oxysporum) 5 种病原菌的抑菌活性, 测试结果如表 2 所示, 在 $10 \mathrm{mg} / \mathrm{L}$ 浓度条件下，化合物 $\mathbf{4 b} 、 \mathbf{4 c} 、 4 \mathbf{d d} 、 \mathbf{4 g} 、 \mathbf{4 h}$ 对除 了黄瓜枯萎病菌之外的 4 种病菌均表现出良好的抑制效 果，其中化合物 4b、4d、4h 对水稻恶苗病菌的抑制率 分别为 $94.42 \% 、 93.42 \% 、 93.75 \%$, 超过了阳性对照氟咯 菌腈的抑制率 $93.33 \%$ ，同时化合物 $\mathbf{4 g}$ 对水稻恶苗病菌 的抑制率也达到了 $89.04 \%$; 化合物 $4 \mathbf{b} 、 4 \mathbf{c} 、 4 \mathbf{4 d} 、 4 \mathrm{~g}$ 、 4h 对水稻纹枯病菌的抑制率分别为 $93.50 \% 、 94.24 \%$ 、 $93.42 \% 、 93.58 \% 、 93.50 \%$ ，均超过了阳性对照氟咯菌腈 的抑制率 $93.33 \%$; 对稻瘟病菌和大豆根腐病菌，化合物 4a $\sim \mathbf{i}$ 均表现出优异的抑菌效果, 其抑制率多数高于阳 性对照的抑制率．在 $1 \mathrm{mg} / \mathrm{L}$ 浓度条件下，化合物 $\mathbf{4 b} 、 4 \mathrm{c} 、$ 4d、4g、4h 对除了黄瓜枯萎病菌之外的 4 种病菌的抑 制率均有所降低，但依然有部分化合物的抑制率与阳性 对照接近, 甚至化合物 $\mathbf{4 g}$ 和 $\mathbf{4 h}$ 对稻瘟病菌的抑制率分 别为 $65.00 \%$ 和 $74.62 \%$, 远高于阳性对照的抑菌率 $54.28 \%$. 化合物 $4 \mathrm{~g}$ 对大豆根腐病菌的抑制率是 $83.56 \%$, 同样高于阳性对照的抑菌率 $77.92 \%$. 证明该系列化合 物具有广谱的抑菌特性, 尤其是化合物 $4 \mathrm{~g}$ 和 $4 \mathrm{~h}$, 均表 现出极佳的抑菌活性, 值得深入研究. 通过文献总结, 我们确认化合物 $4 \mathrm{~g}$ 是未被文献报道过的全新结构，尽 管化合物 $\mathbf{4 a} \sim 4 \mathrm{k}$ 中除化合物 $\mathbf{4 g}$ 外均不是全新结构的化 合物，但它们的生物活性大多数未被文献报道，因此本 文的生物活性测试结果对该系列化合物的开发是一个 重要的补充和说明. 其中化合物 $4 \mathrm{~h}$ 正是杀菌剂拌种咯 的结构, 表现出的优良抑菌效果也再次印证了抑菌活性 测试的真实可靠性.

化合物 5a $\sim 5 \mathbf{j}$ 除了 $5 \mathbf{a} 、 \mathbf{5 b}$ 和 $\mathbf{5 i}$ 外，其他 7 个化合 物均未被文献报道过的全新化合物，从抑菌测试结果来 看, 在 $10 \mathrm{mg} / \mathrm{L}$ 浓度条件下, $\mathbf{5 a} \sim \mathbf{5 j}$ 对除了水稻纹枯病 菌以外的 4 种菌的抑制率均未达到 50\%甚至更低, 但我 们发现对水稻纹枯病菌时，化合物 $5 \mathrm{a} 、 5 \mathrm{~b} 、 5 \mathrm{e} 、 5 \mathrm{~g} 、 5 \mathrm{i}$ 的抑制率可分别达到 $75.55 \% 、 75.07 \% 、 59.85 \%$ 、53.24\%、 $59.81 \%$ ，抑制率超过 50\%甚至达到 75\%的水平，这正是 由于在吡咯环上引入甲基基团，导致化合物的活性发生 显著的变化, 初步证明该系列化合物( $\mathrm{N}$ 位甲基取代的 苯基吡咯类化合物)对水稻纹枯病菌具有一定的专一性, 为我们进一步结构优化提供了方向. 
表 2 苯基吡咯类化合物的抑菌活性测试结果

Table 2 Bactericidal activity test results of the phenylpyrrole compounds

\begin{tabular}{|c|c|c|c|c|c|c|c|c|c|c|}
\hline \multirow[t]{2}{*}{ Compd. } & \multicolumn{2}{|c|}{$\begin{array}{l}\text { Fusarium monili- } \\
\text { forme } \\
\end{array}$} & \multicolumn{2}{|c|}{ Rhizoctonia solani } & \multicolumn{2}{|c|}{$\begin{array}{c}\text { Pyricularia } \\
\text { oryzae }\end{array}$} & \multicolumn{2}{|c|}{$\begin{array}{c}\text { Fusarium oxysporum } \\
\text { f.sp. cucumerinum }\end{array}$} & \multicolumn{2}{|c|}{ Fusarium oxysporu } \\
\hline & $10 \mathrm{mg} \cdot \mathrm{L}^{-}$ & $1 \mathrm{mg} \cdot \mathrm{L}^{-1}$ & $10 \mathrm{mg} \cdot \mathrm{L}^{-}$ & ${ }^{1} 1 \mathrm{mg} \cdot \mathrm{L}^{-1}$ & $10 \mathrm{mg} \cdot \mathrm{L}^{-}$ & $1 \mathrm{mg} \cdot \mathrm{L}^{-1}$ & $10 \mathrm{mg} \cdot \mathrm{L}^{-}$ & $1 \mathrm{mg} \cdot \mathrm{L}^{-1}$ & $10 \mathrm{mg} \cdot \mathrm{L}^{-1}$ & $1 \mathrm{mg} \cdot \mathrm{L}^{-1}$ \\
\hline $4 a$ & 80.01 & 28.78 & 83.75 & 54.76 & 56.71 & 7.46 & 6.94 & 4.16 & 84.61 & 53.84 \\
\hline $4 b$ & 94.42 & 71.71 & 93.50 & 80.51 & 50.00 & 41.3 & 7.04 & 2.11 & 91.55 & 77.27 \\
\hline $4 c$ & 63.88 & 44.22 & 94.24 & 66.82 & 90.71 & 45.71 & 20.39 & 2.63 & 91.42 & 74.28 \\
\hline 4d & 93.42 & 77.63 & 93.42 & 79.6 & 87.85 & 46.42 & 6.08 & 4.72 & 84.86 & 71.71 \\
\hline $4 e$ & 39.22 & 4.71 & 83.93 & 63.6 & 74.26 & 6.61 & 20.25 & 9.49 & 92.14 & 41.42 \\
\hline $4 f$ & 10.49 & 1.01 & 65.27 & 28.43 & 58.33 & 2.08 & 8.02 & 3.08 & 71.23 & 6.16 \\
\hline $4 g$ & 89.04 & 84.24 & 93.58 & 80.12 & 91.42 & 65.00 & 27.84 & 7.59 & 91.78 & 83.56 \\
\hline $4 \mathrm{~h}$ & 93.75 & 90.00 & 93.50 & 72.07 & 79.85 & 74.62 & 48.00 & 13.33 & 64.28 & 12.33 \\
\hline $4 i$ & 72.65 & 36.47 & 56.90 & 23.49 & 77.61 & 38.80 & 19.33 & 5.33 & 88.66 & 52.66 \\
\hline $4 j$ & 10.01 & 0.79 & 20.76 & 1.23 & 0.00 & 0.00 & 7.40 & 2.46 & 6.84 & 4.10 \\
\hline $5 a$ & 13.28 & 0.03 & 75.55 & 57.4 & 0.00 & 0.00 & 9.02 & 5.55 & 28.87 & 2.81 \\
\hline $5 b$ & 32.87 & 1.78 & 75.07 & 46.54 & 17.60 & 6.33 & 9.85 & 4.92 & 32.66 & 17.33 \\
\hline $5 c$ & 32.66 & 3.99 & 47.65 & 8.93 & 21.64 & 8.20 & 12.83 & 3.37 & 29.87 & 16.23 \\
\hline $5 d$ & 19.79 & 1.00 & 35.96 & 3.73 & 25.71 & 15.71 & 16.44 & 10.52 & 31.33 & 13.33 \\
\hline $5 e$ & 18.95 & 1.42 & 59.85 & 12.80 & 21.21 & 6.06 & 12.00 & 6.00 & 15.78 & 5.92 \\
\hline $5 f$ & 10.12 & 0.71 & 22.51 & 0.74 & 15.97 & 13.19 & 10.52 & 1.97 & 7.63 & 4.86 \\
\hline $5 g$ & 17.33 & 5.33 & 53.24 & 20.77 & 13.23 & 2.20 & 7.33 & 4.66 & 16.66 & 9.33 \\
\hline $5 \mathrm{~h}$ & 31.69 & 20.42 & 28.84 & 26.92 & 9.23 & 4.61 & 11.42 & 6.42 & 22.30 & 20.00 \\
\hline $5 \mathbf{i}$ & 15.04 & 1.91 & 59.81 & 25.72 & 36.42 & 7.85 & 25.32 & 12.33 & 38.46 & 7.69 \\
\hline $5 j$ & 8.39 & 0.83 & 22.00 & 2.00 & 4.47 & 1.49 & 5.40 & 3.37 & 15.13 & 4.60 \\
\hline Fludioxonil $^{b}$ & 93.33 & 92.00 & 93.33 & 93.33 & 55.00 & 54.28 & 40.14 & 9.15 & 83.76 & 77.92 \\
\hline
\end{tabular}

${ }^{a}$ The data in the table represents the average value for three replicates of the compound inhibition rate against different bacteria $(\%) .{ }^{b}$ Used as a positive control.

\subsection{CoFMA 模型分析}

三维-定量构效关系(3D-QSAR)技术是经典的药物 设计方法之一, 所建立的三维模型不仅可以借助等势图 揭示出哪些化学结构的改变有利于生物活性的提高, 进 而修饰、优化先导化合物的结构, 同时还可以预测相似 结构化合物的活性. 本文为了开发出新型吡咯类抑菌 剂, 结合第 1.3 节抑菌活性测试结果, 采用比较分子力 场分析 (Comparative molecular force field analysis, CoMFA)方法对目标化合物抑制水稻纹枯病菌活性进行 初步 3D-QSAR 分析，建立了交叉验证系数 $\left(q^{2}\right)$ 为 0.503 、 最佳主成分数 $(N)$ 为 5 、非交叉验证相关系数 $\left(r^{2}\right)$ 为 0.974 、 显著性检验值 $(F)$ 为 81.345、标准估计误差 $(S)$ 为 0.219 的 CoMFA 模型，如表 3 所示. 一般而言, $q^{2}>0.5$ 表明该 模型有很好的预测能力, $r^{2}$ 和 $F$ 值越大, $s$ 值越小, 模型 的拟合能力越好 ${ }^{[24]}$.

表 3 CoMFA 的建模统计学结果

Table 3 Statistical results of CoMFA analysis

\begin{tabular}{|c|c|c|c|c|c|c|}
\hline \multirow{2}{*}{$q^{2 a}$} & \multirow{2}{*}{$N^{b}$} & \multirow{2}{*}{$r^{2 c}$} & \multirow{2}{*}{$S^{d}$} & \multirow{2}{*}{$F^{e}$} & \multicolumn{2}{|c|}{ Contribution $\%$} \\
\hline & & & & & Steric & Electrostatic \\
\hline 0.503 & 5 & 0.974 & 0.219 & 81.345 & 0.542 & 0.458 \\
\hline
\end{tabular}

同时，如表 4 所示给出了化合物 $\mathbf{4 a} \sim \mathbf{5 j}$ 的测试
$\mathrm{pEC}_{50}$ 值和预测 $\mathrm{pEC}_{50}$ 值, 散点图如图 3 所示, 所有数据 拟合较好, $R^{2}$ 为 0.99377 , 这些结果表明该模型是有效 的, 且具有较强的预测能力 ${ }^{[25]}$.

表 4 目标化合物的测试 $\mathrm{pEC}_{50}$ 值与预测 $\mathrm{pEC}_{50}$ 值 ${ }^{a}$

Table 4 Experimental $\mathrm{pEC}_{50}$ value and predicted $\mathrm{pEC}_{50}$ value of target compounds

\begin{tabular}{ccll}
\hline Compd. & Experimental $\mathrm{pEC}_{50}$ & Predicted $\mathrm{pEC}_{50}$ & Residue \\
\hline $\mathbf{4 a}$ & 2.408 & 2.504 & -0.096 \\
$\mathbf{4 b}$ & 3.581 & 3.504 & 0.077 \\
$\mathbf{4 c}$ & 2.774 & 2.691 & 0.083 \\
$\mathbf{4 d}$ & 2.882 & 3.101 & -0.219 \\
$\mathbf{4 e}$ & 2.801 & 2.818 & -0.017 \\
$\mathbf{4} \mathbf{f}^{*}$ & 1.705 & 1.641 & 0.064 \\
$\mathbf{4 g}$ & 3.201 & 3.434 & -0.233 \\
$\mathbf{4 h}$ & 4.876 & 4.693 & 0.183 \\
$\mathbf{4 i}$ & 1.487 & 1.582 & -0.095 \\
$\mathbf{4 j}$ & 0.789 & 0.766 & 0.023 \\
$\mathbf{4 k}$ & 2.950 & 2.912 & 0.038 \\
$\mathbf{5 a}$ & 1.814 & 1.932 & -0.118 \\
$\mathbf{5 b}$ & 2.062 & 2.028 & 0.034 \\
$\mathbf{5 c}$ & 1.890 & 2.039 & -0.149 \\
$\mathbf{5 d}$ & 1.264 & 1.325 & -0.061 \\
$\mathbf{5 e}$ & 1.532 & 1.557 & -0.025 \\
$\mathbf{5 f}$ & 0.847 & 0.697 & 0.150 \\
$\mathbf{5 g}$ & 1.994 & 2.111 & -0.117 \\
$\mathbf{5 i}$ & 1.397 & 1.294 & 0.103 \\
$\mathbf{5 j}$ & 0.735 & 0.820 & -0.085 \\
\hline a & & &
\end{tabular}




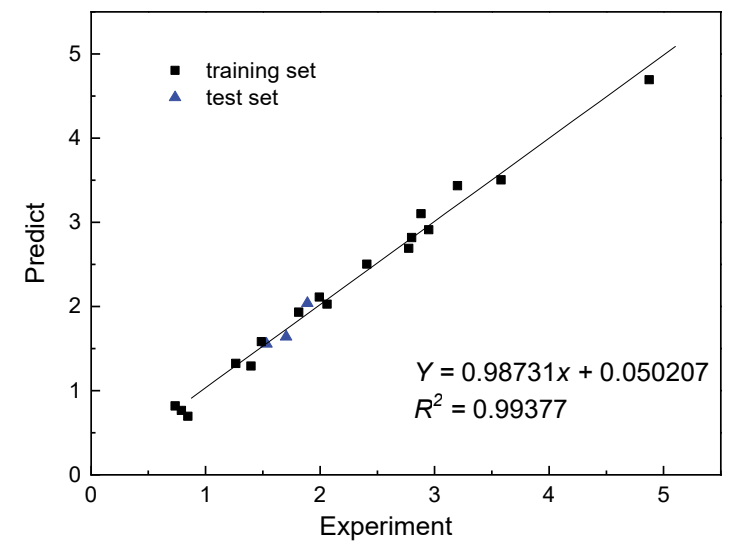

图 3 CoMFA 模型的预测 $\mathrm{pEC}_{50}$ 与实际 $\mathrm{pEC}_{50}$ 的相关性

Figure 3 Predicted $\mathrm{pEC}_{50}$ values of CoMFA model vs. experimental $\mathrm{pEC}_{50}$ values

三维等势图如图 4 所示, 空间场和静电场的贡献率 分别为 $54.2 \%$ 和 $45.8 \%$, 说明二者对该系列化合物活性 均有影响[26]. 绿色和黄色区域代表空间场效应, 减小 黄色区域的空间体积, 增大绿色区域的空间体积有助于 增加化合物的活性, 这提示我们可在苯环的 5 位上增加 取代基团形成与 2 位的对位取代结构, 进而提高抑制活 性; 红色和蓝色区域代表静电场效应, 在三维等势图中 吡咯环的 CN 附近显示红色, 吡咯环与苯环连接部分显 示蓝色, 这与在红色区域引入负电荷基团, 蓝色区域引 入正电荷的取代基有助于增加化合物活性的结论完全 吻合, 这为后续化合物的结构修饰提供重要的指导意 义. 例如, 在苯环上增加取代基团或在吡咯环上的 $\mathrm{N}$ 位 增加芳香基团, 这有待于后续的研究进行验证.

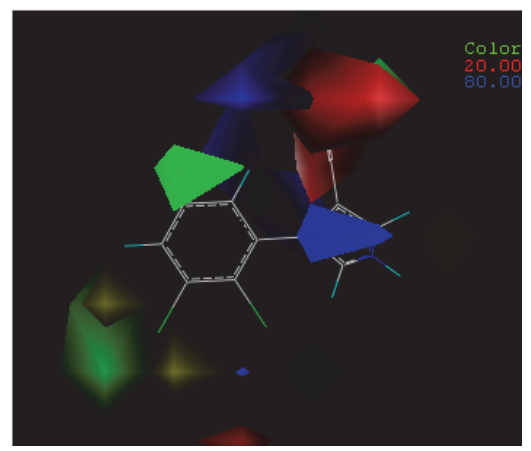

图 4 模型的三维等势图

Figure 4 Three-dimensional equipotential map of the model

\section{2 结论}

以取代的苯甲醛为原料设计合成了 21 个苯基吡咯 类化合物, 对其中 16 个目标化合物进行了单晶结构的 确认. 其中化合物 $4 \mathrm{~g} 、 5 \mathrm{c} \sim 5 \mathrm{~h} 、 5 \mathrm{j}$ 为未被文献报道的全 新结构, 所合成的其他化合物结构虽然不是全新结构, 但是大部分化合物的抑菌活性均未被正式报道. 对 5 种
病原菌生物活性测试结果显示: 在 $10 \mathrm{mg} / \mathrm{L}$ 浓度条件 下，化合物 4b, 4c, 4d, 4g, 4h 对水稻恶苗病菌、水稻纹 枯病菌、稻瘟病菌、大豆根腐病菌均表现出较好甚至高 于阳性对照的抑菌效果; 尤其是化合物 $\mathbf{4 g}$, 在 $1 \mathrm{mg} / \mathrm{L}$ 浓度条件下，对稻瘟病菌和大豆根腐病的抑制率分别是 $65.00 \%$ 和 $83.56 \%$, 高于阳性对照的 $54.28 \%$ 和 $77.92 \%$. 这证明化合物 $4 \mathrm{~g}$ 具有优良的广谱抑菌特性，可作为新 型吡咯类农药的先导化合物进行深入研究. 而 $\mathrm{N}$ 位甲基 取代的化合物只对水稻纹枯病菌表现出专一的抑菌活 性, 达到了探究 $\mathrm{N}$ 位取代基对该类化合物生物活性影响 的目的. 同时, 为了开发出更有效的抗水稻纹枯病菌化 合物，采用 CoMFA 法对 20 个化合物的水稻纹枯病菌活 性进行初步的 3D-QSAR 研究, 建立了一个有效的 CoMFA 模型 $\left(q^{2}=0.503, r^{2}=0.974\right)$, 展现了良好的预测 能力, 三维等势图中所呈现的空间场效应和静电场效应 对该类化合物结构的进一步优化提供了重要的指导意 义.

\section{3 实验部分}

\section{1 仪器与试剂}

Bruker-400 型核磁共振仪 [Bruker, 德国，以 $\mathrm{CDCl}_{3}$ 或二甲基亚砜(DMSO- $\left.d_{6}\right)$ 作溶剂, 三甲硅烷基(TMS)为 内标]; 高效液相色谱仪 LC-20A(岛津, 日本), 傅里叶变 换红外光谱仪-FTIR-8400S(岛津，日本); 高分辨质谱 仪-Q-Exactive (HRMS) Orbitrap(赛默飞, 美国); M70 全 自动熔点仪 (上海佳航, 中国); 元素分析仪 vario MACRO cube (Elementar, 德国). 主要原料为 2 -氟苯甲 醛、2-氯-3-氟苯甲醛等取代的苯甲醛类化合物、其余试 剂均为化学纯或试剂纯.

各化合物纯度由高分辨质谱(HPLC)测试得出, 液 相测试方法: 面积归一化法; 液相色谱分析条件: $\mathrm{C}_{18}$ 反 相硅胶柱, 波长 $230 \mathrm{~nm}$, 流动相为 $V$ (乙腈 $) / V$ (磷酸水, 磷酸的体积分数为 $0.5 \%)=80 / 20$, 流速 $1.0 \mathrm{~mL} / \mathrm{min}$.

\section{2 实验方法}

\subsection{1中间体 2 的合成}

以中间体 $\mathbf{2 c}$ 为例, 在装有温度计、搅拌器、回流冷 凝管的 $250 \mathrm{~mL}$ 四口瓶中装入 $80 \mathrm{~mL}$ 乙醇、 $18.5 \mathrm{~g}(0.1$ mol) 2-溴苯甲醛、 $9.24 \mathrm{~g}(0.11 \mathrm{~mol})$ 氰基乙酰胺和 $5.01 \mathrm{~g}$ (0.11 mol)醋酸胺、0.3 g 三乙基苠基胺盐(TEBA), 加热 回流 $2 \mathrm{~h}$, 然后加入 $100 \mathrm{~mL}$ 水摚拌 $30 \mathrm{~min}$, 过滤析出得 到 E-3-(2-溴苯基)-2-氧基丙烯酰胺(2c) $15.73 \mathrm{~g}$, 白色固 体，纯度 97.5\%, 收率 62.7\%. m.p. 194.4 194.8 ${ }^{\circ} \mathrm{C}$ (lit. $\left.{ }^{[27]} 178.0{ }^{\circ} \mathrm{C}\right) ;{ }^{1} \mathrm{H}$ NMR (400 MHz, DMSO- $\left.d_{6}\right) \delta$ : $7.66 \sim 7.41(\mathrm{~m}, 2 \mathrm{H}), 8.18 \sim 7.68(\mathrm{~m}, 4 \mathrm{H}), 8.30(\mathrm{~s}, 1 \mathrm{H})$. 中 间体 $2 \mathrm{a}, \mathbf{2 b}, \mathbf{2 d} \sim 2 \mathrm{k}$ 的合成步骤与 $\mathbf{2 c}$ 相同. 
E-3-(2-氟苯基)-2-氰基丙烯酰胺(2a): 白色固体, 纯 度为 $97.5 \%$, 收率为 $70.4 \%$ m.p. $138.6 \sim 139.5{ }^{\circ} \mathrm{C}$ (lit. ${ }^{[28]}$ $\left.142 \sim 143{ }^{\circ} \mathrm{C}\right) ;{ }^{1} \mathrm{H}$ NMR (400 MHz, DMSO-d $) \delta: 7.42$ (dd, $J=13.0,5.4 \mathrm{~Hz}, 2 \mathrm{H}), 7.65$ (dd, $J=13.9,7.1 \mathrm{~Hz}$, 1H), 7.95 (d, $J=74.5 \mathrm{~Hz}, 2 \mathrm{H}), 8.09$ (t, $J=7.7 \mathrm{~Hz}, 1 \mathrm{H})$, $8.26(\mathrm{~s}, 1 \mathrm{H})$.

$E$-3-(2-氯苯基)-2-氰基丙烯酰(2b): 白色固体, 纯度 为 $98 \%$, 收率为 $95 \%$. m.p. $164.5 \sim 165.1{ }^{\circ} \mathrm{C}$ (lit. ${ }^{[29]}$ $\left.166 \sim 167{ }^{\circ} \mathrm{C}\right) ;{ }^{1} \mathrm{H}$ NMR (400 MHz, DMSO-d $) \delta: 7.62 \sim$ $7.50(\mathrm{~m}, 2 \mathrm{H}), 7.66(\mathrm{dd}, J=7.9,1.2 \mathrm{~Hz}, 1 \mathrm{H}), 7.99$ (dt, $J=$ $37.2,25.2 \mathrm{~Hz}, 3 \mathrm{H}), 8.36(\mathrm{~s}, 1 \mathrm{H})$.

E-3-(2-(三氟甲基)苯基)-2-氰基丙烯酰胺(2d): 白色 固体，纯度为 $97.2 \%$, 收率为 $40 \%$. m.p. 129.7 $129.8{ }^{\circ} \mathrm{C} ;{ }^{1} \mathrm{H}$ NMR (400 MHz, DMSO- $\left.d_{6}\right) \delta: 7.76(\mathrm{t}, J=$ $7.7 \mathrm{~Hz}, 1 \mathrm{H}), 7.99 \sim 7.81(\mathrm{~m}, 4 \mathrm{H}), 8.07(\mathrm{~s}, 1 \mathrm{H}), 8.41(\mathrm{~d}, J=$ $2.1 \mathrm{~Hz}, 1 \mathrm{H})$. HRMS (ESI) calcd for $\mathrm{C}_{11} \mathrm{H}_{8} \mathrm{~F}_{3} \mathrm{~N}_{2} \mathrm{O}[\mathrm{M}+\mathrm{H}]^{+}$ 241.0583 , found 241.0583 .

E-3-(2-甲基苯基)-2-氭基丙烯酰胺(2e): 白色固体, 纯度为 $98 \%$, 收率为 $80.5 \%$. m.p. $159.9 \sim 160.7{ }^{\circ} \mathrm{C}$ (lit. $\left.{ }^{[28]} 161.0 \sim 161.7{ }^{\circ} \mathrm{C}\right) ;{ }^{1} \mathrm{H}$ NMR (400 MHz, DMSO- $d_{6}$ ) $\delta: 2.38(\mathrm{~s}, 3 \mathrm{H}), 7.40 \sim 7.31(\mathrm{~m}, 2 \mathrm{H}), 7.48 \sim 7.40(\mathrm{~m}, 1 \mathrm{H})$, $7.82(\mathrm{t}, J=13.5 \mathrm{~Hz}, 2 \mathrm{H}), 7.98(\mathrm{~s}, 1 \mathrm{H}), 8.35(\mathrm{~s}, 1 \mathrm{H})$.

E-3-(2-甲氧基苯基)-2-氰基丙烯酰胺(2f): 白色固 体, 纯度为 $97.6 \%$, 收率为 $47 \%$. m.p. $160.3 \sim 160.8{ }^{\circ} \mathrm{C}$ (lit. ${ }^{[30]} 165 \sim 166{ }^{\circ} \mathrm{C}$ ); ${ }^{1} \mathrm{H}$ NMR (400 MHz, DMSO-d $\left.)\right) \delta$ : $3.89(\mathrm{~s}, 3 \mathrm{H}), 7.22 \sim 7.07(\mathrm{~m}, 2 \mathrm{H}), 7.57(\mathrm{t}, J=7.3 \mathrm{~Hz}, 1 \mathrm{H})$, $7.81(\mathrm{~d}, J=54.1 \mathrm{~Hz}, 2 \mathrm{H}), 7.99(\mathrm{~d}, J=7.3 \mathrm{~Hz}, 1 \mathrm{H}), 8.40$ (s, $1 \mathrm{H})$.

E-3-(2-氯-3-氟苯基)-2-氰基丙烯酰胺(2g)：白色固 体, 纯度为 $98 \%$, 收率为 $74.1 \%$. m.p. $158.7 \sim 159.4{ }^{\circ} \mathrm{C}$; ${ }^{1} \mathrm{H}$ NMR (400 MHz, DMSO- $\left.d_{6}\right) \delta: 7.67 \sim 7.55(\mathrm{~m}, 2 \mathrm{H})$, $7.83(\mathrm{~d}, J=6.9 \mathrm{~Hz}, 1 \mathrm{H}), 8.01$ (d, $J=57.8 \mathrm{~Hz}, 2 \mathrm{H}), 8.33$ (s, 1H). HRMS (ESI) calcd for $\mathrm{C}_{10} \mathrm{H}_{7} \mathrm{ClFN}_{2} \mathrm{O}[\mathrm{M}+\mathrm{H}]^{+}$ 225.0225, found 225.0226 .

E-3-(2,3-二氯苯基)-2-氰基丙烯酰胺(2h): 白色固 体, 纯度为 $99.5 \%$, 收率为 $38.4 \%$. m.p. $216.0 \sim 216.5{ }^{\circ} \mathrm{C}$; ${ }^{1} \mathrm{H}$ NMR (400 MHz, DMSO- $\left.d_{6}\right) \delta: 7.57$ (t, $\left.J=8.0 \mathrm{~Hz}, 1 \mathrm{H}\right)$, 7.87 (dd, $J=23.5,7.9 \mathrm{~Hz}, 2 \mathrm{H}), 8.01$ (d, $J=59.2 \mathrm{~Hz}, 2 \mathrm{H})$, $8.35(\mathrm{~s}, 1 \mathrm{H})$. HRMS (ESI) calcd for $\mathrm{C}_{10} \mathrm{H}_{7} \mathrm{Cl}_{2} \mathrm{~N}_{2} \mathrm{O}[\mathrm{M}+$ $\mathrm{H}]^{+}$240.9930, found 240.9930 .

E-3-(2,3-二甲基苯基)-2-氰基丙烯酰胺(2i): 白色固 体, 纯度为 $97.7 \%$, 收率为 $30 \%$. m.p. 169.0 169.2 ${ }^{\circ} \mathrm{C}$; ${ }^{1} \mathrm{H}$ NMR (400 MHz, DMSO-d $) \delta: 2.25$ (s, 3H), 2.29 (s, $3 \mathrm{H}), 7.23$ (t, $J=7.7 \mathrm{~Hz}, 1 \mathrm{H}), 7.33$ (d, $J=7.4 \mathrm{~Hz}, 1 \mathrm{H}), 7.56$ (d, $J=7.7 \mathrm{~Hz}, 1 \mathrm{H}), 7.88$ (d, $J=85.0 \mathrm{~Hz}, 2 \mathrm{H}), 8.42$ (s, 1H).
HRMS (ESI) calcd for $\mathrm{C}_{12} \mathrm{H}_{13} \mathrm{~N}_{2} \mathrm{O}[\mathrm{M}+\mathrm{H}]^{+}$201.1022, found 201.1022 .

E-3-(2,3-二甲氧基苯基)-2-氭基丙烯酰胺(2j): 浅黄 色固体，纯度为 $98.7 \%$, 收率为 $30.9 \%$. m.p. 174.4 $175.1{ }^{\circ} \mathrm{C} ;{ }^{1} \mathrm{H}$ NMR (400 MHz, DMSO- $\left.d_{6}\right) \delta: 3.81(\mathrm{~s}, 3 \mathrm{H})$, $3.86(\mathrm{~s}, 3 \mathrm{H}), 7.34 \sim 7.19(\mathrm{~m}, 2 \mathrm{H}), 7.61(\mathrm{dd}, J=7.8,1.1 \mathrm{~Hz}$, 1H), 7.86 (d, $J=70.7 \mathrm{~Hz}, 2 \mathrm{H}), 8.33$ (s, 1H). HRMS (ESI) calcd for $\mathrm{C}_{12} \mathrm{H}_{13} \mathrm{~N}_{2} \mathrm{O}_{3}[\mathrm{M}+\mathrm{H}]^{+}$233.0921, found 233.0919 .

E-3-(2-氟-3-(三氟甲基)苯基)-2-氰基丙烯酰胺(2k): 白色固体，纯度为 $98 \%$, 收率为 $35 \%$. m.p. 154.0 $154.6{ }^{\circ} \mathrm{C}$; ${ }^{1} \mathrm{H}$ NMR (400 MHz, DMSO- $\left.d_{6}\right) \delta: 7.62$ (t, $J=$ $7.9 \mathrm{~Hz}, 1 \mathrm{H}), 8.17 \sim 7.85(\mathrm{~m}, 3 \mathrm{H}), 8.27(\mathrm{~s}, 1 \mathrm{H}), 8.33$ (t, $J=$ $7.2 \mathrm{~Hz}, 1 \mathrm{H})$. HRMS (ESI) calcd for $\mathrm{C}_{11} \mathrm{H}_{7} \mathrm{~F}_{4} \mathrm{~N}_{2} \mathrm{O}[\mathrm{M}+\mathrm{H}]^{+}$ 259.0489 , found 259.0489 .

3.2 .2 目标化合物 $\mathbf{4 a} \sim \mathbf{4 k}$ 的合成

中间体 3 的合成方法参照文献[16]且表征数据与文 献一致.

以目标化合物 $\mathbf{4 c}$ 为例, 在装有温度计、搅拌器、回 流冷凝管、滴液漏斗的 $250 \mathrm{~mL}$ 的四口瓶中装入 $50 \mathrm{~mL}$ 甲醇，加入中间体 2c $3.95 \mathrm{~g}(0.016 \mathrm{~mol})$ 和 $3.1 \mathrm{~g}(0.016$ $\mathrm{mol}$ )中间体 3 (TosMIC), 在冰浴的条件下滴加由 $20 \mathrm{~mL}$ 甲醇和 $1.79 \mathrm{~g}(0.032 \mathrm{~mol}) \mathrm{KOH}$ 组成的混合液, 在室温 下反应 $3 \mathrm{~h}$ 后减压蒸出甲醇, 加入 $50 \mathrm{~mL}$ 水搅拌 $0.5 \mathrm{~h}$, 过滤干燥后用甲醇重结晶得到白固体粉末 4-(2-溴苯 基)- $1 H$-吡咯-3-腈(4c)：2.97 g, 纯度 $96.8 \%$, 收率 75.2\%. m.p. 139.4 140.5 ${ }^{\circ} \mathrm{C} ;{ }^{1} \mathrm{H}$ NMR (400 MHz, DMSO- $\left.d_{6}\right) \delta$ : $7.10(\mathrm{~s}, 1 \mathrm{H}), 7.26 \sim 7.32(\mathrm{~m}, 1 \mathrm{H}), 7.37 \sim 7.447(\mathrm{~m}, 2 \mathrm{H})$, $7.66 \sim 7.77(\mathrm{~m}, 2 \mathrm{H}), 11.93(\mathrm{~s}, 1 \mathrm{H}) ;{ }^{13} \mathrm{C}$ NMR $(101 \mathrm{MHz}$, DMSO- $\left.d_{6}\right) \delta$ : 134.01 (s), 132.96 (s), 131.89 (s), 129.27 (s), 127.71 (s), 127.19 (s), 124.43 (s), 122.99 (s), 119.49 (s), 116.63 (s), 92.14 (s); IR (KBr) v: 3278, 2220, 1452, 1027, 754, $749 \mathrm{~cm}^{-1}$. Anal. calcd for $\mathrm{C}_{11} \mathrm{H}_{7} \mathrm{BrN}_{2}$ : C 53.47, $\mathrm{H}$ 2.86, N 11.34; found C 53.41, H 2.83, N 11.22. 目标化合 物 $4 a 、 4 b 、 4 d \sim 4 k$ 的合成步骤与 $4 \mathrm{c}$ 相同.

4-(2-氟苯基)-1H-吡咯-3-腈(4a)：白色固体粉末，纯 度 98\%, 收率 98\%. m.p. 104.2 104.5 ${ }^{\circ} \mathrm{C} ;{ }^{1} \mathrm{H}$ NMR (400 MHz, DMSO-d $d_{6} \delta: 7.21(\mathrm{~d}, J=1.7 \mathrm{~Hz}, 1 \mathrm{H}), 7.24 \sim 7.39$ $(\mathrm{m}, 3 \mathrm{H}), 7.62(\mathrm{td}, J=7.7,1.5 \mathrm{~Hz}, 1 \mathrm{H}), 7.74(\mathrm{~s}, 1 \mathrm{H}), 12.01$ $(\mathrm{s}, 1 \mathrm{H}) ;{ }^{13} \mathrm{C}$ NMR $\left(101 \mathrm{MHz}, \mathrm{DMSO}-d_{6}\right) \delta: 158.83$ (d, $J_{\mathrm{C}-\mathrm{F}}$ $=246.1 \mathrm{~Hz}), 129.48(\mathrm{~d}, J=3.5 \mathrm{~Hz}), 128.71(\mathrm{~d}, J=8.2 \mathrm{~Hz})$, 128.34 (s), 124.66 (d, $J=3.5 \mathrm{~Hz}), 120.88$ (d, $J=14.3 \mathrm{~Hz}$ ), 119.89 (d, $J=6.5 \mathrm{~Hz}), 118.38$ (s), 116.96 (s), 115.94 (d, $J=22.2 \mathrm{~Hz}$ ), 90.76 (s); IR (KBr) v: 3274, 2223, 1463, $1220,814,744 \mathrm{~cm}^{-1}$. Anal. calcd for $\mathrm{C}_{11} \mathrm{H}_{7} \mathrm{FN}_{2}$ : C 70.96, 
H 3.79, N 15.05; found C 70.48, H 3.66, N 15.55.

4-(2-氯苯基)- $1 H$-吡咯-3-腈(4b): 白色固体粉末, 纯 度 98.5\%, 收率 75.1\%. m.p. 130.5 $131.2{ }^{\circ} \mathrm{C} ;{ }^{1} \mathrm{H} \mathrm{NMR}$ $\left(400 \mathrm{MHz}, \mathrm{DMSO}-d_{6}\right) \delta: 7.14(\mathrm{~s}, 1 \mathrm{H}), 7.28 \sim 7.60(\mathrm{~m}, 4 \mathrm{H})$, $7.71(\mathrm{~s}, 1 \mathrm{H}), 11.96$ (s, 1H); ${ }^{13} \mathrm{C}$ NMR (101 MHz, DMSO$\left.d_{6}\right) \delta: 132.14(\mathrm{~s}), 131.92(\mathrm{~s}), 131.65(\mathrm{~s}), 129.83(\mathrm{~s}), 128.96$ (s), 127.44 (s), 127.24 (s), 122.49 (s), 119.75 (s), 116.69 (s), 92.04 (s); IR (KBr) v: 3283, 2220, 1455, 1085, 749, $729 \mathrm{~cm}^{-1}$. Anal. calcd for $\mathrm{C}_{11} \mathrm{H}_{7} \mathrm{ClN}_{2}$ : C 65.20, H 3.48, N 13.82; found $\mathrm{C} 65.25, \mathrm{H} 3.66, \mathrm{~N} 13.55$.

4-(2-(三氟甲基)苯基)- $1 H$-吡咯-3-腈(4d): 白色固体 粉末, 纯度 $98 \%$, 收率 50\%. m.p. 100.7 102.5 ${ }^{\circ} \mathrm{C} ;{ }^{1} \mathrm{H}$ NMR (400 MHz, DMSO- $\left.d_{6}\right) \delta: 6.98(\mathrm{~s}, 1 \mathrm{H}), 7.46$ (d, $J=$ $7.6 \mathrm{~Hz}, 1 \mathrm{H}), 7.59$ (t, $J=7.7 \mathrm{~Hz}, 1 \mathrm{H}), 7.74 \sim 7.68(\mathrm{~m}, 2 \mathrm{H})$, $7.82(\mathrm{~d}, J=7.8 \mathrm{~Hz}, 1 \mathrm{H}), 11.92(\mathrm{~s}, 1 \mathrm{H}) ;{ }^{13} \mathrm{C} \mathrm{NMR}(101$ $\left.\mathrm{MHz}, \mathrm{DMSO}-d_{6}\right) \delta$ : 133.07 (s), 132.17 (s), 128.12 (s), 127.78 (s), 127.05 (s), 126.04 (q, $J=5.6 \mathrm{~Hz}), 125.40$ (s), $122.68(\mathrm{~s}), 122.39(\mathrm{~s}), 119.28\left(\mathrm{~d}, J_{\mathrm{C}-\mathrm{F}}=2.8 \mathrm{~Hz}\right), 116.38(\mathrm{~s})$, 92.64 (s); IR (KBr) v: 3285, 2222, 1313, 1035, 770, 755 $\mathrm{cm}^{-1}$. Anal. calcd for $\mathrm{C}_{12} \mathrm{H}_{7} \mathrm{~F}_{3} \mathrm{~N}_{2}$ : C 60.61, H 3.00, $\mathrm{N}$ 11.71; found C 61.02, H 2.99, N 11.86.

4-(2-甲苯基)- $1 H$-吡咯-3-腈(4e): 白色固体粉末, 纯 度 $98 \%$, 收率 46\%. m.p. 114.3 115.2 ${ }^{\circ} \mathrm{C} ;{ }^{1} \mathrm{H}$ NMR (400 MHz, DMSO-d6) $\delta: 2.29(\mathrm{~s}, 3 \mathrm{H}), 7.01(\mathrm{t}, J=2.2 \mathrm{~Hz}, 1 \mathrm{H})$, $7.16 \sim 7.34(\mathrm{~m}, 4 \mathrm{H}), 7.60 \sim 7.76(\mathrm{~m}, 1 \mathrm{H}), 11.86(\mathrm{~s}, 1 \mathrm{H})$; ${ }^{13} \mathrm{C}$ NMR (101 MHz, DMSO-d $\left.d_{6}\right) \delta: 135.79(\mathrm{~s}), 132.74(\mathrm{~s})$, 130.26 (s), 130.01 (s), 127.21 (s), 127.19 (s), 127.16 (s), 125.71 (s), 125.07 (s), 118.78 (s), 117.17 (s), 91.84 (s), 20.22 (s); IR (KBr) v: 3248, 2224, 1523, 1088, 764, 753 $\mathrm{cm}^{-1}$. Anal. calcd for $\mathrm{C}_{12} \mathrm{H}_{10} \mathrm{~N}_{2}$ : C 79.10, H 5.53, N 15.37; found $\mathrm{C} 79.00, \mathrm{H} 5.74, \mathrm{~N} 15.21$.

4-(2-甲氧基苯基)- $1 H$-吡咯-3-腈(4f): 白色固体粉 末, 纯度 $97.7 \%$, 收率 41.9\%. m.p. 134.9 135.2 ${ }^{\circ} \mathrm{C} ;{ }^{1} \mathrm{H}$ NMR (400 MHz, DMSO- $\left.d_{6}\right) \delta: 3.79(\mathrm{~s}, 3 \mathrm{H}), 6.98(\mathrm{td}, J=$ $7.5,0.9 \mathrm{~Hz}, 1 \mathrm{H}), 7.0 \sim 7.15(\mathrm{~m}, 2 \mathrm{H}), 7.22 \sim 7.31(\mathrm{~m}, 1 \mathrm{H})$, $7.43(\mathrm{dd}, J=7.5,1.6 \mathrm{~Hz}, 1 \mathrm{H}), 7.56 \sim 7.70(\mathrm{~m}, 1 \mathrm{H}), 11.78$ $(\mathrm{s}, 1 \mathrm{H}) ;{ }^{13} \mathrm{C}$ NMR (101 MHz, DMSO- $\left.d_{6}\right) \delta$ : $156.07(\mathrm{~s})$, 129.17 (s), 128.19 (s), 127.30 (s), 121.93 (s), 121.29 (s), 120.42 (s), 119.41 (s), 117.48 (s), 111.48 (s), 91.37 (s), 55.21 (s); IR (KBr) v: 3260, 2216, 1523, 1025, 792, 760 $\mathrm{cm}^{-1}$. Anal. calcd for $\mathrm{C}_{12} \mathrm{H}_{10} \mathrm{~N}_{2} \mathrm{O}$ : C 72.71, $\mathrm{H}$ 5.09, N 14.13; found C 72.11, H 5.24, N 13.86.

4-(2-氯-3-氟苯基)- $1 H$-吡咯-3-腈(4g): 白色固体粉 末, 纯度 $97 \%$, 收率 $87.5 \%$. m.p. $134.0 \sim 134.5{ }^{\circ} \mathrm{C} ;{ }^{1} \mathrm{H}$ NMR (400 MHz, DMSO- $\left.d_{6}\right) \delta: 7.21(\mathrm{~d}, J=2.0 \mathrm{~Hz}, 1 \mathrm{H})$,
$7.27 \sim 7.34(\mathrm{~m}, 1 \mathrm{H}), 7.36 \sim 7.48(\mathrm{~m}, 2 \mathrm{H}), 7.75(\mathrm{~d}, J=2.0$ $\mathrm{Hz}, 1 \mathrm{H}), 12.05(\mathrm{~s}, 1 \mathrm{H}) ;{ }^{13} \mathrm{C}$ NMR (101 MHz, DMSO- $\left.d_{6}\right) \delta$ : $157.90\left(\mathrm{~d}, J_{\mathrm{C}-\mathrm{F}}=245.7 \mathrm{~Hz}\right), 134.26(\mathrm{~s}), 128.31(\mathrm{~d}, J=8.8$ $\mathrm{Hz}), 127.76$ (s), 127.03 (d, $J=2.8 \mathrm{~Hz}), 121.20$ (s), 120.17 (s), 118.96 (d, $J=17.4 \mathrm{~Hz}), 116.48$ (s), 115.48 (d, $J=21.6$ $\mathrm{Hz}$, 91.97 (s); IR (KBr) v: 3284, 2224, 1443, 1086, 795, $770 \mathrm{~cm}^{-1}$. Anal. calcd for $\mathrm{C}_{11} \mathrm{H}_{6} \mathrm{ClFN}_{2}$ : C 59.88, H 2.74, N 12.70; found C 59.05, H 2.72, N 12.38.

4-(2,3-二氯苯基)- $1 H$-吡咯-3-腈(4h): 白色固体粉 末, 纯度 $99.5 \%$, 收率 78.4\%. m.p. 150.0 150.3 ${ }^{\circ} \mathrm{C}$ (lit. $\left.{ }^{[31]} 152 \sim 153{ }^{\circ} \mathrm{C}\right) ;{ }^{1} \mathrm{H}$ NMR (400 MHz, DMSO-d $\left.d_{6}\right) \delta$ : $7.19(\mathrm{~d}, J=1.7 \mathrm{~Hz}, 1 \mathrm{H}), 7.37 \sim 7.45(\mathrm{~m}, 2 \mathrm{H}), 7.60 \sim 7.66$ $(\mathrm{m}, 1 \mathrm{H}), 7.74(\mathrm{~d}, J=1.6 \mathrm{~Hz}, 1 \mathrm{H}), 12.04(\mathrm{~s}, 1 \mathrm{H}) ;{ }^{13} \mathrm{C} \mathrm{NMR}$ (101 MHz, DMSO-d $) \delta$ : 134.45 (s), 132.38 (s), 130.43 (s), 130.35 (s), 129.55 (s), 128.10 (s), 127.57 (s), 122.32 (s), 120.08 (s), 116.46 (s), 92.06 (s); IR (KBr) v: 3298, 2224, $1459,1086,782,765 \mathrm{~cm}^{-1}$. Anal. calcd for $\mathrm{C}_{11} \mathrm{H}_{6} \mathrm{Cl}_{2} \mathrm{~N}_{2}$ : C 55.73, H 2.55, N 11.82; found C 55.56, H 2.52, N 11.72.

4-(2,3-二甲苯基)-1H-吡咯-3-腈(4i)：白色固体粉末， 纯度 $98.2 \%$, 收率 77.6\% m.p. 150.9 $151.7{ }^{\circ} \mathrm{C} ;{ }^{1} \mathrm{H}$ NMR $\left(400 \mathrm{MHz}, \mathrm{DMSO}-d_{6}\right) \delta: 2.17(\mathrm{~s}, 3 \mathrm{H}), 2.28(\mathrm{~s}, 3 \mathrm{H}), 6.92(\mathrm{~s}$, $1 \mathrm{H}), 7.10$ (ddd, $J=15.1,9.7,6.6 \mathrm{~Hz}, 3 \mathrm{H}), 7.67$ (s, 1H), $11.83(\mathrm{~s}, 1 \mathrm{H}) ;{ }^{13} \mathrm{C}$ NMR (101 MHz, DMSO- $\left.d_{6}\right) \delta: 136.74$ (s), 134.58 (s), 132.90 (s), 128.81 (s), 128.08 (s), 126.93 (s), 125.91 (s), 125.14 (s), 118.63 (s), 117.11 (s), 92.24 (s), 20.35 (s), 16.55 (s); IR (KBr) v: 3302, 2225, 1518, 1146, $744,727 \mathrm{~cm}^{-1}$. Anal. calcd for $\mathrm{C}_{13} \mathrm{H}_{12} \mathrm{~N}_{2}$ : C 79.56, H 6.16, N 14.27; found C 78.58, H 6.35, N 13.95.

4-(2,3-二甲氧基苯基)- $1 H$-吡咯-3-腈(4j): 白色固体 粉末, 纯度 $96 \%$, 收率 $26.5 \%$. m.p. $122.9 \sim 123.5{ }^{\circ} \mathrm{C} ;{ }^{1} \mathrm{H}$ NMR (400 MHz, DMSO- $\left.d_{6}\right) \delta: 3.61$ (s, 3H), 3.83 (s, 3H), $7.00(\mathrm{dd}, J=6.4,3.3 \mathrm{~Hz}, 1 \mathrm{H}), 7.06 \sim 7.13(\mathrm{~m}, 2 \mathrm{H}), 7.15(\mathrm{~d}$, $J=1.6 \mathrm{~Hz}, 1 \mathrm{H}), 7.67(\mathrm{~d}, J=1.3 \mathrm{~Hz}, 1 \mathrm{H}), 11.85(\mathrm{~s}, 1 \mathrm{H}) ;{ }^{13} \mathrm{C}$ NMR (101 MHz, DMSO-d6) $\delta: 152.94$ (s), 145.87 (s), 130.16 (s), 128.90 (s), 127.31 (s), 123.93 (s), 120.89 (s), 119.40 (s), 117.38 (s), 111.75 (s), 91.11 (s), 59.68 (s), 55.73 (s); IR (KBr) v: 3306, 2219, 1470, 1002, 791, 745 $\mathrm{cm}^{-1}$. Anal. calcd for $\mathrm{C}_{13} \mathrm{H}_{12} \mathrm{~N}_{2} \mathrm{O}_{2}$ : C 68.41, H 5.30, N 12.27; found C 67.62, H 5.42, N 12.08.

4-(2-氟-3-(三氟甲基)苯基)- $1 H$-吡咯-3-腈(4k): 白色 固体粉末，纯度 $98.3 \%$, 收率 55.6\%. m.p. 144.3 $144.6{ }^{\circ} \mathrm{C} ;{ }^{1} \mathrm{H}$ NMR (400 MHz, DMSO- $\left.d_{6}\right) \delta: 7.34(\mathrm{~s}, 1 \mathrm{H})$, 7.48 (t, $J=7.8 \mathrm{~Hz}, 1 \mathrm{H}), 7.71(\mathrm{t}, J=7.0 \mathrm{~Hz}, 1 \mathrm{H}), 7.80$ (s, $1 \mathrm{H}), 7.93$ (t, $J=7.3 \mathrm{~Hz}, 1 \mathrm{H}), 12.15(\mathrm{~s}, 1 \mathrm{H}) ;{ }^{13} \mathrm{C} \mathrm{NMR}(101$ $\left.\mathrm{MHz}, \mathrm{DMSO}-d_{6}\right) \delta: 155.56\left(\mathrm{~d}, J_{\mathrm{C}-\mathrm{F}}=255.1 \mathrm{~Hz}\right), 134.25(\mathrm{~s})$, 
128.73 (s), 125.55 (d, $J=4.3 \mathrm{~Hz}), 125.11$ (d, $J=4.3 \mathrm{~Hz})$, 124.09 (s), 122.61 (d, $J=13.5 \mathrm{~Hz}), 121.39$ (s), 120.65 (d, $J=6.4 \mathrm{~Hz}), 117.41\left(\mathrm{dd}, J_{\mathrm{C}-\mathrm{F}}=32.0,12.8 \mathrm{~Hz}\right), 116.61(\mathrm{~d}$, $J=21.7 \mathrm{~Hz}$ ), 90.95 (s); IR (KBr) v: 3254, 2232, 1530, $1146,784,738 \mathrm{~cm}^{-1}$. Anal. calcd for $\mathrm{C}_{13} \mathrm{H}_{12} \mathrm{~N}_{2} \mathrm{O}_{2}$ : C 56.70, H 2.38, N 11.02; found C 56.30, H 2.40, N 10.96 .

\subsection{3 目标化合物 $\mathbf{5 a} \sim \mathbf{5} \mathbf{j}$ 的合成}

在装有温度计、搅拌器、空气冷凝管、滴液漏斗的

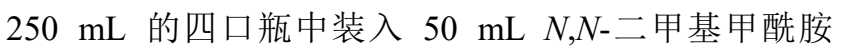
(DMF), 加入 4-(2-澳苯基)- $1 H$-吡咯-3-腈(5c) $2.47 \mathrm{~g} \mathrm{(0.01}$ $\mathrm{mol}), 1.1 \mathrm{~g}(0.0075 \mathrm{~mol})$ 碳酸钾, 冰浴 $10{ }^{\circ} \mathrm{C}$ 下滴加 2.84 $\mathrm{g}(0.02 \mathrm{~mol})$ 碘甲烷, 滴加完在室温条件下反应 $1 \mathrm{~h}$ 后减 压蒸出大部分 $\mathrm{DMF}$, 加入 $50 \mathrm{~mL}$ 水搅拌 $0.5 \mathrm{~h}$, 过滤干燥 后用甲醇重结晶得到白固体粉末 4-(2-溴苯基)-1-甲基吡咯-3-腈 $(\mathbf{5 c}) 1.95 \mathrm{~g}$, 纯度 97\%, 收率 74.7\%. m.p. 66.9 68.3 ${ }^{\circ} \mathrm{C}$; ${ }^{1} \mathrm{H}$ NMR (400 MHz, DMSO- $d_{6}$ ) $\delta: 3.72$ (s, $3 \mathrm{H}), 7.11(\mathrm{~d}, J=2.2 \mathrm{~Hz}, 1 \mathrm{H}), 7.29(\mathrm{td}, J=7.9,1.9 \mathrm{~Hz}, 1 \mathrm{H})$, 7.42 (dtd, $J=9.4,7.6,1.4 \mathrm{~Hz}, 2 \mathrm{H}), 7.66$ (d, $J=2.2 \mathrm{~Hz}$, $1 \mathrm{H}), 7.73(\mathrm{dd}, J=8.0,0.8 \mathrm{~Hz}, 1 \mathrm{H}) ;{ }^{13} \mathrm{C}$ NMR $(101 \mathrm{MHz}$, DMSO- $\left.d_{6}\right) \delta$ : $133.66(\mathrm{~s}), 133.00(\mathrm{~s}), 131.77(\mathrm{~s}), 130.10(\mathrm{~s})$, 129.39 (s), 127.76 (s), 124.81 (s), 123.22 (s), 122.82 (s), 116.21 (s), 91.86 (s), 36.49 (s); IR (KBr) v: 3132, 2223, $1529,1155,811,743 \mathrm{~cm}^{-1}$. Anal. calcd for $\mathrm{C}_{12} \mathrm{H}_{9} \mathrm{FN}_{2}$ : C 55.20, H 3.47, N 10.73; found C 54.85, H 3.43, N 10.57 . 目标化合物 $5 \mathbf{a} 、 5 \mathbf{b} 、 5 \mathrm{~d} \sim \mathbf{5 j}$ 的合成步骤与 $\mathbf{5 c}$ 相同.

4-(2-氟苯基)-1-甲基-吡咯-3-腈(5a): 白色固体粉末， 纯度 $97.5 \%$, 收率 $60.5 \%$. m.p. $75.2 \sim 76.2{ }^{\circ} \mathrm{C} ;{ }^{1} \mathrm{H}$ NMR (400 MHz, DMSO- $\left.d_{6}\right) \delta: 3.73(\mathrm{~s}, 3 \mathrm{H}), 7.22(\mathrm{t}, J=2.1 \mathrm{~Hz}$, $1 \mathrm{H}), 7.24 \sim 7.40(\mathrm{~m}, 3 \mathrm{H}), 7.61$ (td, $J=7.8,1.7 \mathrm{~Hz}, 1 \mathrm{H})$, $7.71(\mathrm{~d}, J=2.2 \mathrm{~Hz}, 1 \mathrm{H}) ;{ }^{13} \mathrm{C}$ NMR $\left(101 \mathrm{MHz}\right.$, DMSO- $\left.d_{6}\right)$ $\delta: 158.73$ (d, $J=246.3 \mathrm{~Hz}$ ), 131.17 (s), 129.22 (d, $J=3.4$ $\mathrm{Hz}), 128.85$ (d, $J=8.3 \mathrm{~Hz}), 124.71$ (d, $J=3.4 \mathrm{~Hz}), 123.58$ (d, $J=6.9 \mathrm{~Hz}), 120.49$ (d, $J=14.1 \mathrm{~Hz}), 118.67$ (s), 116.51 (s), 115.98 (d, $J=22.1 \mathrm{~Hz}$ ), 90.42 (s), 36.53 (s); IR (KBr) v: 3128, 2218, 1492, 1152, 820, $777 \mathrm{~cm}^{-1}$. Anal. calcd for $\mathrm{C}_{12} \mathrm{H}_{9} \mathrm{FN}_{2}$ : C 71.99, H 4.53, N 13.99; found C 70.06, H 4.59, N 13.39 .

4-(2-氯苯基)-1-甲基-吡咯-3-腈(5b): 白色固体粉末， 纯度 98.6\%, 收率 66.4\%. m.p. 85.5 86.7 ${ }^{\circ} \mathrm{C} ;{ }^{1} \mathrm{H}$ NMR (400 MHz, DMSO- $\left.d_{6}\right) \delta: 3.72(\mathrm{~s}, 3 \mathrm{H}), 7.15(\mathrm{~d}, J=2.1 \mathrm{~Hz}$, 1H), 7.39 (dqd, $J=14.0,7.3,2.3 \mathrm{~Hz}, 3 \mathrm{H}$ ), $7.53 \sim 7.58$ (m, 1H), $7.68(\mathrm{~d}, J=2.1 \mathrm{~Hz}, 1 \mathrm{H}) ;{ }^{13} \mathrm{C}$ NMR $(101 \mathrm{MHz}$, DMSO- $\left.d_{6}\right) \delta: 131.99(\mathrm{~s}), 131.55(\mathrm{~s}), 131.48(\mathrm{~s}), 130.34(\mathrm{~s})$, 129.86 (s), 129.06 (s), 127.28 (s), 123.49 (s), 122.81 (s), 116.27 (s), 91.71 (s), 36.51 (s); IR (KBr) v: 3131, 2212,
1471, 1158, 815, $763 \mathrm{~cm}^{-1}$. Anal. calcd for $\mathrm{C}_{12} \mathrm{H}_{9} \mathrm{ClN}_{2}$ : C 66.52, H 4.19, N 12.93; found C 66.35, H 4.29, N 12.78.

4-(2-(三氟甲基)苯基)-1-甲基-吡咯-3-腈(5d): 白色 固体粉末, 纯度 $97 \%$, 收率 $69.2 \%$. m.p. $96.9 \sim 98.1{ }^{\circ} \mathrm{C}$; ${ }^{1} \mathrm{H}$ NMR (400 MHz, DMSO- $d_{6}$ ) $\delta: 3.72$ (s, 3H), 6.99 (s, 1H), $7.44(\mathrm{~d}, J=7.6 \mathrm{~Hz}, 1 \mathrm{H}), 7.60(\mathrm{t}, J=7.7 \mathrm{~Hz}, 1 \mathrm{H}), 7.67$ (d, $J=2.1 \mathrm{~Hz}, 1 \mathrm{H}), 7.72$ (t, $J=7.6 \mathrm{~Hz}, 1 \mathrm{H}), 7.83$ (d, $J=7.7$ $\mathrm{Hz}, 1 \mathrm{H}) ;{ }^{13} \mathrm{C}$ NMR (101 MHz, DMSO- $\left.d_{6}\right) \delta: 132.99$ (s), 132.23 (s), 131.71 (s), 129.98 (s), 128.24 (s), 127.81 (d, $J=28.9 \mathrm{~Hz}), 126.06$ (q, $\left.J_{\mathrm{C}-\mathrm{F}}=5.3 \mathrm{~Hz}\right), 125.36(\mathrm{~s}), 123.05$ (d, $J=2.4 \mathrm{~Hz}), 122.73$ (d, $J=20.0 \mathrm{~Hz}), 115.96$ (s), 92.34 (s), 36.46 (s); IR (KBr) v: 3127, 2219, 1510, 1131, 818, $772 \mathrm{~cm}^{-1}$. Anal. calcd for $\mathrm{C}_{13} \mathrm{H}_{9} \mathrm{~F}_{3} \mathrm{~N}_{2}: \mathrm{C} 62.40, \mathrm{H} 3.63, \mathrm{~N}$ 11.20; found C 62.07, H 3.69, N 11.02.

4-(2-甲苯基)-1-甲基-吡咯-3-腈(5e)：白色固体粉末， 纯度 $98 \%$, 收率 $46 \%$. m.p. $76.6 \sim 78.2{ }^{\circ} \mathrm{C}$ (lit. ${ }^{[32]} 87 \sim$ $\left.89{ }^{\circ} \mathrm{C}\right) ;{ }^{1} \mathrm{H}$ NMR (400 MHz, DMSO- $\left.d_{6}\right) \delta: 2.29$ (s, 3H), 7.01 (t, $J=2.2 \mathrm{~Hz}, 1 \mathrm{H}), 7.16 \sim 7.34(\mathrm{~m}, 4 \mathrm{H}), 7.60 \sim 7.76$ (m, 1H), $11.86(\mathrm{~s}, 1 \mathrm{H}) ;{ }^{13} \mathrm{C}$ NMR (100 MHz, $\left.\mathrm{CDCl}_{3}\right) \delta$ : 136.88 (s), 130.02 (s), 129.90 (s), 129.54 (s), 127.74 (s), 126.31 (s), 119.89 (s), 116.93 (s), 91.11 (s), 36.95 (s), 21.17 (s); IR (KBr) v: 3128, 2214, 1524, 1153, 821, 756 $\mathrm{cm}^{-1}$. Anal. calcd for $\mathrm{C}_{12} \mathrm{H}_{10} \mathrm{~N}_{2}$ : C 79.56, H 6.16, N 14.27; found C 79.50, H 6.37, N 14.16.

4-(2-甲氧基苯基)-1-甲基-吡咯-3-腈(5f): 白色固体 粉末, 纯度 $97.7 \%$, 收率 $40 \%$. m.p. 83.3 84.0 ${ }^{\circ} \mathrm{C} ;{ }^{1} \mathrm{H}$ NMR (400 MHz, DMSO- $\left.d_{6}\right) \delta$ : 3.69 (s, 3H), 3.80 (s, 3H), $6.98(\mathrm{t}, J=7.2 \mathrm{~Hz}, 1 \mathrm{H}), 7.10(\mathrm{dd}, J=13.4,5.2 \mathrm{~Hz}, 2 \mathrm{H})$, $7.25 \sim 7.32(\mathrm{~m}, 1 \mathrm{H}), 7.43(\mathrm{dd}, J=7.5,1.6 \mathrm{~Hz}, 1 \mathrm{H}), 7.60(\mathrm{~d}$, $J=2.2 \mathrm{~Hz}, 1 \mathrm{H}) ;{ }^{13} \mathrm{C}$ NMR $\left(101 \mathrm{MHz}\right.$, DMSO- $\left.d_{6}\right) \delta: 156.00$ (s), $130.23(\mathrm{~s}), 128.96(\mathrm{~s}), 128.32$ (s), 123.29 (s), 121.68 (s), 121.55 (s), 120.43 (s), 117.05 (s), 111.51 (s), 91.03 (s), 55.22 (s), 36.39 (s); IR (KBr) v: 3129, 2214, 1494, 1155, $813,764 \mathrm{~cm}^{-1}$. Anal. calcd for $\mathrm{C}_{13} \mathrm{H}_{12} \mathrm{~N}_{2} \mathrm{O}: \mathrm{C} 73.56, \mathrm{H}$ 5.70, N 13.20; found C 73.07, H 5.82, N 13.01.

4-(2-氯-3-氟苯基)-1-甲基-吡咯-3-腈(5g): 白色固体 粉末, 纯度 $97.9 \%$, 收率 $63.4 \%$. m.p. $163.9 \sim 164.8{ }^{\circ} \mathrm{C}$; ${ }^{1} \mathrm{H}$ NMR (400 MHz, DMSO- $\left.d_{6}\right) \delta: 3.73(\mathrm{~s}, 3 \mathrm{H}), 7.22(\mathrm{~d}$, $J=2.2 \mathrm{~Hz}, 1 \mathrm{H}), 7.30(\mathrm{dd}, J=5.0,3.3 \mathrm{~Hz}, 1 \mathrm{H}), 7.37 \sim 7.50$ $(\mathrm{m}, 2 \mathrm{H}), 7.72(\mathrm{~d}, J=2.2 \mathrm{~Hz}, 1 \mathrm{H}) ;{ }^{13} \mathrm{C}$ NMR $(101 \mathrm{MHz}$, DMSO- $\left.d_{6}\right) \delta: 157.90\left(\mathrm{~d}, J_{\mathrm{C}-\mathrm{F}}=245.8 \mathrm{~Hz}\right), 133.87(\mathrm{~s})$, 130.66 (s), 128.38 (d, $J=8.7 \mathrm{~Hz}), 126.86$ (d, $J=3.0 \mathrm{~Hz}$ ), 123.87 (s), 121.51 (s), 118.83 (d, $J=17.3 \mathrm{~Hz}), 116.07$ (s), 115.60 (d, $J=21.5 \mathrm{~Hz}), 91.65$ (s), 36.57 (s); IR (KBr) v: $3131,2221,1448,1156,812,788 \mathrm{~cm}^{-1}$. Anal. calcd for 
$\mathrm{C}_{12} \mathrm{H}_{8} \mathrm{ClFN}_{2}$ : C 61.42, H 3.44, N 11.94; found $\mathrm{C} 61.41, \mathrm{H}$ $3.48, \mathrm{~N} 11.84$.

4-(2,3-二氯苯基)-1-甲基-吡咯-3-腈(5h): 白色固体 粉末, 纯度 $99.5 \%$, 收率 94.7\%. m.p. 189.5 190.4 ${ }^{\circ} \mathrm{C}$; ${ }^{1} \mathrm{H}$ NMR (400 MHz, DMSO-d $\left.d_{6}\right) \delta: 3.73$ (s, 3H), 7.19 (d, $J=2.0 \mathrm{~Hz}, 1 \mathrm{H}), 7.37 \sim 7.45(\mathrm{~m}, 2 \mathrm{H}), 7.64(\mathrm{dd}, J=7.6,1.8$ $\mathrm{Hz}, 1 \mathrm{H}), 7.71(\mathrm{~d}, J=2.0 \mathrm{~Hz}, 1 \mathrm{H}) ;{ }^{13} \mathrm{C} \mathrm{NMR}(101 \mathrm{MHz}$, DMSO-d $\left.d_{6}\right) \delta: 134.06(\mathrm{~s}), 132.43$ (s), 130.47 (s), 130.30 (s), 130.19 (s), 129.65 (s), 128.16 (s), 123.77 (s), 122.60 (s), 116.05 (s), 91.76 (s), 36.55 (s); IR (KBr) v: 3131, 2221, 1411, 1161, 813, $791 \mathrm{~cm}^{-1}$. Anal. calcd for $\mathrm{C}_{12} \mathrm{H}_{8} \mathrm{Cl}_{2} \mathrm{~N}_{2}$ : C 57.40, H 3.21, N 11.16; found C 57.52 H 3.37, N 10.92.

4-(2,3-二甲苯基)-1-甲基-吡咯-3-腈(5i): 白色固体 粉末, 纯度 $97.1 \%$, 收率 $20 \%$. m.p. 98.7 99.8 ${ }^{\circ} \mathrm{C} ;{ }^{1} \mathrm{H}$ NMR (400 MHz, DMSO-d $\left.d_{6}\right) \delta: 2.17$ (s, 3H), 2.28 (s, 3H), 3.71 (s, 3H), 6.92 (d, $J=2.2 \mathrm{~Hz}, 1 \mathrm{H}), 7.04$ (d, $J=7.3 \mathrm{~Hz}$, $1 \mathrm{H}), 7.07 \sim 7.18(\mathrm{~m}, 2 \mathrm{H}), 7.63(\mathrm{~d}, J=2.2 \mathrm{~Hz}, 1 \mathrm{H}) ;{ }^{13} \mathrm{C}$ NMR (101 MHz, DMSO-d $d_{6} \delta: 136.82$ (s), 134.47 (s), 132.56 (s), 129.85 (s), 128.91 (s), 127.93 (s), 126.39 (s), 125.18 (s), 122.50 (s), 116.70 (s), 91.89 (s), 36.40 (s), 20.32 (s), 16.53 (s); IR (KBr) v: 3127, 2213, 1523, 1156, $816,798 \mathrm{~cm}^{-1}$. Anal. calcd for $\mathrm{C}_{14} \mathrm{H}_{14} \mathrm{~N}_{2}$ : C 79.97, H 6.71, N 13.32; found C 79.66, H 6.92, N 13.15.

4-(2,3-二甲氧基苯基)-1-甲基-吡咯-3-腈(5j): 白色 固体粉末, 纯度 $96.7 \%$, 收率 53.3\%. m.p. 79.1 89.6 ${ }^{\circ} \mathrm{C}$; ${ }^{1} \mathrm{H}$ NMR (400 MHz, DMSO-d $)_{6} \delta: 3.62(\mathrm{~s}, 3 \mathrm{H}), 3.71$ (s, $3 \mathrm{H}), 3.83(\mathrm{~s}, 3 \mathrm{H}), 7.00(\mathrm{dd}, J=7.5,2.2 \mathrm{~Hz}, 1 \mathrm{H}), 7.03 \sim$ $7.12(\mathrm{~m}, 2 \mathrm{H}), 7.14(\mathrm{~d}, J=2.2 \mathrm{~Hz}, 1 \mathrm{H}), 7.64$ (d, $J=2.2 \mathrm{~Hz}$, $1 \mathrm{H}) ;{ }^{13} \mathrm{C}$ NMR (101 MHz, DMSO- $d_{6}$ ) $\delta: 152.93$ (s), 145.80 (s), 130.53 (s), 126.65 (s), 123.95 (s), 123.27 (s), 121.14 (s), 120.82 (s), 116.97 (s), 111.86 (s), 90.79 (s), 59.76 (s), 55.72 (s), 36.45 (s); IR (KBr) v: 3129, 2218, 1524, 1108, $799,756 \mathrm{~cm}^{-1}$. Anal. calcd for $\mathrm{C}_{14} \mathrm{H}_{14} \mathrm{~N}_{2} \mathrm{O}_{2}$ : C 69.41, H 5.82, N 11.56; found C 69.14, H 5.98, N 11.46.

\subsection{4 目标化合物的抑菌活性测试}

采用生长速率法对目标化合物进行抑菌活性测 试 ${ }^{[29]}$. 以水稻恶苗病菌(Fusarium moniliforme)、水稻纹 枯病菌(Rhizoctonia solani)、稻瘟病菌(Pyricularia oryzae)、黄瓜枯萎病菌(Fusarium oxysporum f.sp. cucumerinum) 和大豆根腐病菌(Fusarium oxysporum) 5 种病 原菌为测试菌种. 在无菌条件下以二甲基亚砜 (DMSO- $\left.d_{6}\right)$ 为溶剂, 将各待测化合物配置成相应浓度的 药液备用. 选择各待测化合物对病原菌产生最大抑制率 $\geqslant 50 \%$ 的 8 个浓度梯度, 将待测化合物药液定量加入已 灭菌的 $\operatorname{PDA}($ 马铃薯-葡萄糖培养基)中, 充分混合均匀
后，倒入灭菌的培养血中制成含药平板，每个处理重复 3 次. 用直径 $5 \mathrm{~mm}$ 的打孔器在培养 $4 \mathrm{~d}$ 的待测试菌种边 缘取新鲜菌碟接种于上述含药平板中, 在 $(27 \pm 2){ }^{\circ} \mathrm{C}$ 的 恒温培养箱中培养, 以加入等量 $\mathrm{DMSO}-d_{6}$ 的培养基为 空白对照. 采用十字交叉法测量菌落直径, 并计算出菌 丝生长抑制率, 然后使用 Origin 2018 软件进行数据处 理, 计算出各目标化合物的有效中浓度 $\mathrm{EC}_{50}$ 值(表 4)和 相关系数 $(r)$.

抑制率 $(\%)=($ 对照菌落扩展直径一处理菌落扩展

直径) $/$ (对照菌落扩展直径) $\times 100 \%$

\subsubsection{CoMFA 模型的建立}

将合成的 20 个目标化合物用于 3D-QSAR 研究, 并 将实验测得的水稻纹枯病菌的 $\mathrm{EC}_{50}$ 值转化为 $\mathrm{pEC}_{50}$ 值 $\left(-\log \mathrm{EC}_{50}\right)^{[34]}$. 数据集所有的分子结构、 $\mathrm{EC}_{50}$ 值以及 $\mathrm{pEC}_{50}$ 值, 如表 4 所示. 首先使用 ChemDraw 19.0 绘制化 合物结构, 导入 Sybyl-x 2.0 中并利用 Minimize 模块以分 子力学对化合物三维结构优化, 能量收玫标准 4.184 $\mathrm{kJ} / \mathrm{mol}$, 最大迭代次数 10000 次, 在标准 Tripos 力场下, 负载 Gasteiger-Huckel 电荷, 采用 Powell 能量梯度法优 化.

研究采用 CoMFA 方法建立模型, 将 20 个化合物按 照个数比 5:1 原则分为训练集(Training set)和测试集 (Test set). 其中测试集 4 个分子在表 4 中以“*”表示, 含 9 号模板分子(一般选取样本中生物活性最强的分子)用 于验证模型. 然后采用 Align database 模块, 基于 9 号模 板分子的公共骨架, 分别对训练集和测试集进行分子叠 合，选取的模板分子和公共骨架相同.

辅助材料(Supporting Information) 所合成的目标化 合物 ${ }^{1} \mathrm{H}$ NMR 谱图、 ${ }^{13} \mathrm{C}$ NMR 谱图、FTIR 谱图、单分 子结构图和相关参数. 这些材料可以免费从本刊网站 (http://sioc-journal.cn/)上下载.

\section{References}

[1] Vitaku, E.; Smith, D. T.; Njardarson, J. T. J. Med. Chem. 2014, 57, 10257.

[2] Sony, M. M. S.; Ponnuswamy, M. N. Cryst. Growth Des. 2006, 6, 736.

[3] Chen, X.-T.; Chen, J.; Ke, J.-J.; Zhang, K.; Wu, P.-P.; Wang, S.-H. Chin. J. Org. Chem. 2021, 41, 206 (in Chinese). (陈学涛, 陈洁, 柯俊杰, 张焜, 吴盼盼, 王少华, 有机化学, 2021, 41, 206.)

[4] Bulumulla, C.; Gunawardhana, R.; Gamage, P. L.; Miller, J. T.; Kularatne, R. N.; Biewer, M. C.; Stefan, M. C. ACS Appl. Mater. Inter. 2020, 12, 32209.

[5] Petri, G. L.; Spanò, V.; Spatola, R.; Holl, R.; Raimondi, M. V.; Barraja, P.; Montalbano, A. Eur. J. Med. Chem. 2020, 202, 112783.

[6] Xu, S.-C.; Jiang, M.-G. Chin. J. Pestic. Sci. 2002, 4, 2 (in Chinese). (徐尚成, 蒋木庚, 农药学学报, 2002, 4, 2.)

[7] Han, F.-G.; Lu, Y.; Ji, X.-M.; Zhao, M.-Q.; Zhang, X.-Y.; Liu, Y. Chin. J. Org. Chem. 2010, 30, 1080 (in Chinese). 
(韩富根, 卢叶, 姬小明, 赵铭钦, 张晓蕴, 刘云, 有机化学, 2010, 30, 1080.)

[8] Wang, M.-Z.; Xu, H.; Feng, Q.; Wang, L.-Z.; Wang, S.-H.; Li, Z.-M. J. Agric. Food Chem. 2009, 57, 7912.

[9] Yang, Y.-Z.; Jiao, B.-N. Modern Agrochem. 2007, 6, 5 (in Chinese). (杨玉柱，焦必宁，现代农药, 2009, 6, 5.)

[10] Li, C.; Liao, D.-H.; Zeng, Z.-W.; Wang, M.-J.; Wu, Z.-X.; Yang, F.; Shi, W.-J. Modern Agrochem. 2009, 8, 3 (in Chinese). (李超, 廖道华, 曾仲武, 王美娟, 吴忠信, 杨芳, 师文娟, 现代 农药, 2009, 8, 3.)

[11] Zhao, Y.-H.; Wang, Q.-H.; Wang, Y.; Zhang, Z.-Q; Wei, Y.; Liu, F.; Zhou, C.-G.; Mu, W. J. Agric. Food Chem. 2017, 65, 5908.

[12] Cheng, Y.-N.; Xie, G.-Y.; Sun, S.-J.; You, X.-F. Agrochemicals 2010, 49,8 (in Chinese).

(程绎南，谢桂英，孙淑君，游秀峰，农药, 2010, 49, 8.)

[13] Tseng, C. K.; Gless Jr, R. D. J. Org. Chem. 1983, 48, 3566.

[14] Zhang, M.-J.; Chen, S.-J.; Weng, Z.-Q. Org. Lett. 2018, $20,481$.

[15] Nosachev, S. B.; Tyrkova, E. A.; Tyrkov. A. G. Russ. J. Org. Chem. 2009, 45, 624

[16] Wang, Z.-S.; Ye, H.-T.; Xiao, D.; Ma, D.-S.; Gao, J.-S.; Yu, Y.-H.; Hou, G.-F.; Yan, P.-F. Chin. J. Org. Chem. 2014, 34, 2057 (in Chinese).

(王子时, 叶汉蹈, 肖丹, 马东升, 高金胜, 于颖慧, 侯广峰, 间 鹏飞，有机化学, 2014, 34, 2057.)

[17] Wu, Q.; J, J.; Liu, C.-L.; Yang, J.-C. Agrochemicals 2012, 51, 4 (in Chinese).

(吴峤, 焦姣, 刘长令, 杨吉春, 农药, 2012, 51, 4.)

[18] Wu, C.-C.; Yu, X.-B.; Wang, B.-L.; Liu, J.-B.; Meng, F.-F.; Zhao, Y.-Y.; Xiong, L.-X.; Yang, N.; Li, Y.-X.; Li, Z. M. J. Agric. Food Chem. 2020, 68, 9319.

[19] Yao, T.-T. Ph.D. Dissertation, Zhejiang University, Hangzhou, 2017 (in Chinese) (姚婷婷, 博士论文, 浙江大学, 杭州, 2017.)

[20] Li, Y.; Zhang, H.-Q.; Liu, J.; Yang, X.-P.; Liu, Z.-J. J. Agric. Food
Chem. 2006, 54, 3636

[21] Chen, Q.-Q.; Huggins, M.-T.; Lightner, D.-A.; Norona, W.; McDonagh, A.-F. J. Am. Chem. Soc. 1999, 121, 9253.

[22] Santo, R. D.; Tafi, A.; Costi, R.; Botta, M.; Artico, M.; Corelli, F.; Forte, M.; Caporuscio, F.; Angiolella, L.; Palamare, A. T. J. Med. Chem. 2005, 48, 5140 .

[23] Zhu, R.; Xing, L.-X.; Liu, Y.-T.; Deng, F.-K.; Wang, X.-Y.; Hu, Y.-F. J. Org. Chem. 2008, 693, 3897.

[24] Yu, Y.-P.; Duan, W.-G.; Lin, G.-S.; Kang, G.-Q.; Wang, X.-Y.; Lei, F.-H. Chin. J. Org. Chem. 2020, 40, 1647 (in Chinese). (虞友培, 段文贵, 林桂汕, 康国强, 王晓宇, 雷福厚, 有机化学, 2020, 40, 1647.)

[25] Yan Y.-L.; Li, Y.; Zhang, S.-W.; Ai, C.-Z. J. Mol. Graph. Model. 2011, 29, 747.

[26] Zeng, G.-H.; Fang, D.-Q.; Wu, W.-J.; Zhang, R.; Xie, W.-G.; Wu, J.-H.; Shen, Y. Int. J. Quantum. Chem. 2013, 113, 1467.

[27] Rupanwar, B. D.; Chavan, S. S.; Shelke, A. M.; Suryavanshi, G. M. New J. Chem. 2018, 42, 6433.

[28] Silver, R. F.; Kerr, K. A.; Frandsen, P. D.; Kelley, S. J.; Holmes, H. L. Can. J. Chem. 1967, 45, 1001.

[29] Wang, D.-Y.; Xi, G.-H.; Ma, J.-J.; Wang, C.; Zhang, X.-C.; Wang, Q.-Q. Synth. Commun. 2011, 41, 3060

[30] Bezgin, D. A.; Ershov, O. V.; Ievlev, M. Y.; Belikov, M. Y.; Bardasov, I. N. Russ. J. Org. Chem. 2018, 54, 1100.

[31] Van Leusen, D.; Van Echten, E.; M. Van Leusen, A. J. Org. Chem. 1992, 57, 2245.

[32] Prasad, P. V.; Shanker, M.; Venkanna, A.; Swamy, M. K.; Gopichand, K.; Rao, P. V. Synth. Commun. 2018, 48, 1040

[33] Yao, T.-T.; Xiao, D.-X.; Li, Z.-S.; Cheng, J.-L.; Fang, S.-W.; Du, Y.-J.; Zhao, J.-H.; Dong, X.-W.; Zhu, G.-N. J. Agric. Food. Chem. 2017, 65, 5397.

[34] Huang, S.-S.; Ren, Y.-J.; Peng, X.-X.; Qian, P.-P.; Meng, L.-W. Eur. J. Pharm. Sci. 2019, 137, 104965. 